\title{
Implementación de control PID de nivel en laboratorio usando PLC Siemens S7-300
}

\section{Level PID control lab implementation using PLC Siemens S7-300}

\author{
Armando Simmonds-Mendoza ${ }^{1}$, Nehider Cabrera-Londoño², Neimir Berdugo-Barandica ${ }^{3}$, Javier Roldán- \\ Mckinley $^{4}$, Eugenio Yime-Rodríguez ${ }^{5}$

\begin{abstract}
${ }^{1}$ Ingeniería Mecánico, Universidad del Atlántico, Colombia. Email: simns07@ hotmail.com ${ }^{2}$ Ingeniería Mecánico, Universidad del Atlántico, Colombia. Email: gloster_gladiattor@hotmail.com

${ }^{3}$ Ingeniería Mecánico, Universidad del Atlántico, Colombia. Email: neimir_19@hotmail.com

${ }^{4}$ Grupo de investigación en Diseño de Sistemas Mecánicos y Robóticos para la Producción Industria, Ingeniería Mecánica, Universidad del Atlántico, Colombia. Email: javierroldan@mail.uniatlantico.edu.co

${ }^{5}$ Grupo de investigación en Diseño de Sistemas Mecánicos y Robóticos para la Producción Industria, Ingeniería Mecánica, Universidad del Atlántico, Colombia. Email: eugenioyime@mail.uniatlantico.edu.co
\end{abstract}

Recibido: mayo 24, 2017. Aceptado: marzo 10, 2018. Versión final: abril 14, 2018.

\section{Resumen}

Se detalla la implementación de un controlador PID didáctico para el control de nivel de agua. El hardware integra una bomba centrífuga, tuberías y elementos conectores varios, válvulas de flujo, una válvula proporcional DANFOSS, un sensor ultrasónico de nivel FLOWLINE DL10 y usando Simatic WinCC en un PLC Siemens S7-300. Los parámetros del proceso fueron determinados experimentalmente y utilizados para calcular los valores de las ganancias PID ante una entrada tipo escalón. Se dedujo la ecuación ideal del proceso, usada como planta en un modelo del sistema ideal en Simulink, y su respuesta fue comparada con la experimental. Bajo condiciones de apreciables cambios en la consigna, el control tipo proporcional $\mathrm{P}$ muestra el mejor desempeño representado en un rápido tiempo de respuesta. El tipo de control PID mostró ser ventajoso cuando se trata de mantener la variable controlada cerca al punto de consigna, o de seguir pequeños cambios en el set point; la abertura de la válvula requiere un poco más de tiempo para set points relativamente grandes.

Palabras clave: Control de nivel; PID; S7-300; instrumentación industrial.

\begin{abstract}
A didactic PID controller implementation for water level control is detailed. Hardware is comprised a comprised of pump, pipelines and various connecting elements, flow valves, a DANFOSS proportional valve, a FLOWLINE DL10 ultrasound level sensor and Simatic WinCC in a Siemens S7300 PLC. Experimental tests allowed estimating process parameters, that were used to calculate the control gains before a unit step forcing function. The deduced ideal process equation was used as the plant of a Simulink model to compare against experimental findings. Under moderate changes in the set point, proportional $(\mathrm{P})$ control exhibits the best performance with a rapid response time. PID control was advantageous when the control variable is near the set point (small changes) since the valve opening requires more time for relatively big set points.
\end{abstract}

ISSN Impreso: 1657 - 4583, ISSN en línea: 2145 - 8456

Este artículo puede compartirse bajo la licencia CC BY-ND 4.0 y se referencia usando el siguiente formato: A. Simmonds-Mendoza, N. CabreraLondoño, N. Berdugo-Barandica, J. Roldán-Mckinley, E. Yime-Rodríguez, "Implementación de control PID de nivel en laboratorio usando PLC Siemens S7-300,” Rev. UIS Ing., vol. 17 no. 2, pp. 159-178, 2018. Doi: https://doi.org/10.18273/revuin.v17n2-2018015 
Keywords: Level control; PID; S7-300; industrial instrumentation.

\section{Introducción}

El dominio del control automático de procesos es parte de las competencias profesionales del ingeniero mecánico; este debe ser capaz de analizar, modelar, diseñar y evaluar componentes y sistemas básicos de control automático [1]; asimismo, el control automático ha desempeñado una función vital en el avance de la ingeniería y la ciencia [2], por lo cual los fundamentos teóricos sistemas y su integración con los controladores lógicos programables (PLC) son esenciales para el desarrollo posterior de una buena praxis.

El control por retroalimentación es la estrategia de control más común en la industria [3], en donde se pretende que cada día los procesos sean más automatizados y con el mayor control posible que minimice los costos de operación y mantenimiento; pero a la vez, que brinde altos estándares de calidad. A su vez, dentro del control por realimentación, la estrategia basada en el algoritmo PID (Proporcional, Integral, Derivativo) se ha venido usando desde un poco antes de mediados del siglo XX, cuando Taylor Instruments introdujo al mercado en 1940 el primer controlador PID [4]. Desde entonces, se utilizan ampliamente en toda la industria, donde proporcionan un rendimiento satisfactorio y robusto para una variedad de procesos, [5], [6], [7], [8].

El control de nivel ocupa un lugar importante en la industria química y de alimentos, razón por la cual se encuentran documentados numerosos trabajos en los ámbitos académico e industrial, para facilitar el entendimiento tanto de la estrategia de control como la aplicación a la variable nivel de líquido. En la Universidad Rodrigo Facio de Costa Rica, por ejemplo, se desarrolló la implementación de un controlador PID en un microcontrolador ATmega16, para el control de diferentes variables de proceso [9], entre ellas el nivel como variable de interés; además de la aplicación de algoritmos PID para programar PLC [10]. En Venezuela se implementa un PLC Telemecanique TSX Micro 3721001 para control PID de diferentes variables, entre ellas la variable de nivel, utilizando Matlab para la etapa de simulación [11]. También en Suramérica, en Ecuador, se le dedica importancia a esta temática en una tesis de pregrado que combina lógica difusa dentro de control por realimentación usando LabVIEW y una tarjeta NI-DAQ 6008 [12] para aplicaciones académicas.
En Colombia también se han desarrollado trabajos que apuntan al fortalecimiento de la academia para entender y explicar adecuadamente el proceso de control de nivel. En el año 2004 se desarrolló en la Universidad de los Andes un sistema de control de multivariable, incluida la variable de nivel [13], que posteriormente permitió la implementación de LabVIEW 9.1 en esa planta multipropósito [14]. Por su parte, Gil y Robayo, en la U. Tecnológica de Pereira, implementaron un sistema de almacenamiento y control de nivel de líquido como modelo de segundo orden, usando un controlador SIPART DR-20 [15]. Más recientemente, se implementa el control de nivel en dos tanques interconectados a nivel de laboratorio en la Universidad Industrial de Santander, empleando un controlador industrial Honeywell UDC1200 con modelado detallado de las capacitancias y resistencias hidráulicas del proceso [16], con posterior sintonización del controlador PID. Siendo el control de nivel un tópico de la teoría de Control de mucha aplicación en la industria, se hace pertinente que los laboratorios de ingeniería de la Universidad del Atlántico cuenten con un banco especializado que permita el refuerzo de los conceptos y el uso de dispositivos electrónicos actuales, que permita la futura comparación de diversas estrategias para control de nivel de tanque como se realizó en [17], o inclusive el control de nivel en el tanque de manera remota a través del internet [18]. Un trabajo reciente en el área de control y modelado de sistemas térmicos con control PID también es reportado por Fuentes et al. [19], en el cual se integra un sistema embebido para control PID, mediante señal de modulación de ancho de pulso.

Este artículo integra los conceptos teóricos y la implementación de una planta con la instrumentación necesaria para práctica de laboratorio de control de nivel de agua, por medio de la programación de la rutina de proceso y configuración del algoritmo PID en el PLC S7300; y así aplicar las técnicas de control para estimar los parámetros iniciales que requiere el PLC, y determinar cuál acción de control presenta mejor desempeño en el proceso de control de nivel. Se inicia con el modelado dinámico del proceso y elementos (válvula y sensor) que permite hallar la función de transferencia del proceso. Se continúa con la aplicación de la prueba escalón al sistema, y la posterior comparación de resultados teóricos y experimentales que permitan determinar los valores de las ganancias del controlador PID. La interacción de banco de nivel con el PLC S7300 se facilita con una interfaz gráfica creada en Simatic-SCADA. 


\section{Metodología}

\subsection{Modelado dinámico}

Un sistema de control es un conjunto de dispositivos encargados de administrar, ordenar, dirigir o regular el comportamiento de otro sistema (o subsistema), también llamado planta dentro del sistema de control. Un sistema de control se implementa con el fin de controlar máquinas o actuadores, buscando reducir las probabilidades de fallo y mantener ciertos valores de variables a intervalos determinados, típicos de procesos de producción industriales para controlar equipos o máquinas. Los sistemas de control realimentados, véase figura 1, se denominan también sistemas de control en lazo cerrado, y se caracterizan porque un elemento llamado controlador es alimentado con el error, e, que es calculado mediante la comparación de la variable de referencia, R, y la variable real o de proceso $\mathrm{Y}$.

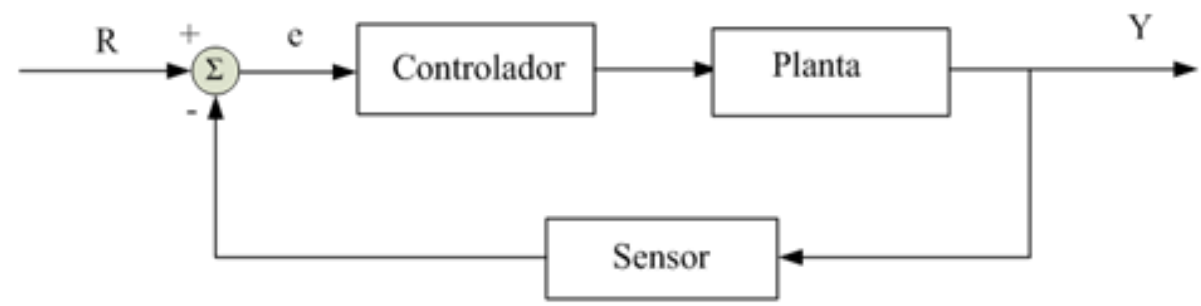

Figura 1. Sistema de control en lazo cerrado. Fuente: elaboración propia.

La generación de la señal de error, e, requiere de un sensor y de un arreglo electrónico para comparar y amplificar las señal de referencia y de proceso. El elemento controlador incluye los componentes electrónicos y el actuador del proceso, el cual genera el cambio en la variable de salida hasta ser ajustada a la de referencia. La importancia de estos componentes estriba en que realizan las tres operaciones obligatorias básicas que deben estar presentes en todo sistema de control; estas operaciones son: Medición (M): la medición de la variable que se controla se hace generalmente mediante la combinación de sensor y transmisor; Decisión (D): con base en la medición, el controlador decide qué hacer para mantener la variable en el valor que se desea; Acción (A): como resultado de la decisión del controlador se debe efectuar una acción en el sistema, generalmente esta es realizada por el elemento final de control.

\subsubsection{Resistencia y capacitancia en sistema de nivel de líquido}

Considere el sistema que aparece en la figura 2 en el cual se integra un tanque con líquido, una válvula de control a la entrada y una válvula a la salida del depósito que actúa como válvula de carga. Controlar el nivel de líquido en el tanque implica conocimiento del comportamiento matemático de cada elemento y su integración en una ecuación global. El flujo de líquido a través de un tubo corto debe vencer la resistencia $\mathrm{R}$ de sus paredes, la cual depende del acabado interno, el diámetro y del material. $\mathrm{Si}$ ese tubo corto conecta dos tanques, la resistencia de ese tramo está asociada al cambio en la diferencia de nivel de líquido entre los dos tanques. El conocimiento de la resistencia, $\mathrm{R}$, permite relacionar el cambio en la diferencia de nivel, $\Delta \mathrm{h}$, con el cambio en el caudal del fluido, $\Delta \mathrm{Q}$, a través del tramo de tubería conectando los tanques, es decir:

$$
R=\frac{\Delta h}{\Delta Q}
$$

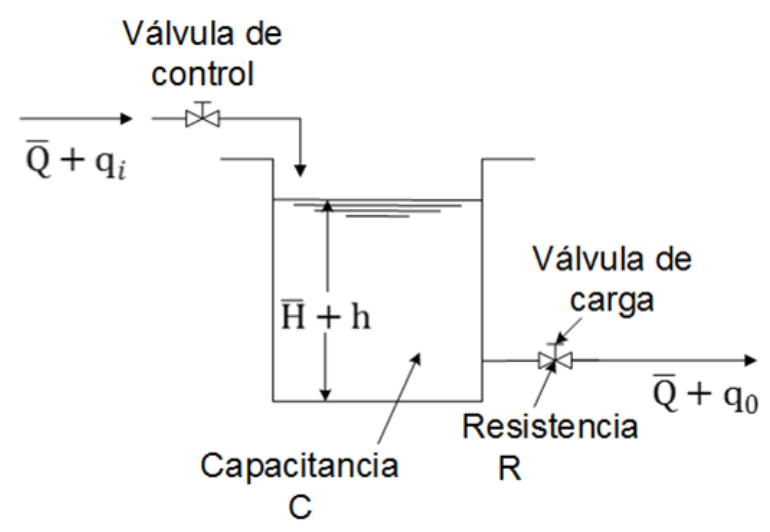

Figura 2. Sistema de nivel de líquido. Fuente: elaboración propia.

Dado que la relación entre la velocidad del flujo y la diferencia de nivel es distinta para flujo laminar y flujo turbulento, en lo sucesivo se consideran ambos casos. $\mathrm{Si}$ el fluido que sale a través de la válvula de carga en el sistema de la figura 2 fluye en régimen laminar [20] (número de Reynolds<2000), la relación entre la velocidad del flujo en estado estable y la altura en estado estable en el nivel de la restricción se obtiene mediante (2).

$$
Q=K H,
$$


Donde Q: caudal del flujo del líquido en estado estable $\left[\mathrm{m}^{3} / \mathrm{s}\right] ; \mathrm{K}$ : coeficiente de área del sistema $\left[\mathrm{m}^{2} / \mathrm{s}\right]$, y $\mathrm{H}$ : altura en estado estable $[\mathrm{m}]$. La resistencia de flujo laminar constante $\mathrm{R}_{\mathrm{L}}$ se obtiene con (3).

$$
R_{L}=\frac{d H}{d Q}=\frac{H}{Q}
$$

Si el flujo es turbulento a través de la restricción (válvula de carga en la figura 2), entonces la velocidad del flujo en estado estable se obtiene mediante (4).

$$
Q=K \sqrt{H} \text {. }
$$

La resistencia $R_{T}$ para el flujo turbulento se obtiene mediante la ecuación (5). Al reemplazar la ecuación (4) en la (5), y despejando se obtiene el diferencial de caudal en la ecuación (6), y $\mathrm{R}_{\mathrm{T}}$ se expresa como en la ecuación (7).

$$
\begin{aligned}
& R_{T}=\frac{d H}{d Q} \\
& d Q=\frac{K}{2 \sqrt{H}} d H \\
& R_{T}=\frac{2 H}{Q}
\end{aligned}
$$

El valor de la resistencia de flujo turbulento $R_{T}$ depende del flujo y la altura. Sin embargo, el valor de $\mathrm{R}_{\mathrm{T}}$ se considera constante si los cambios en la altura y en el flujo son pequeños. Usando la resistencia de flujo turbulento, la relación entre $\mathrm{Q}$ y $\mathrm{H}$ se obtiene mediante (8).

$$
Q=\frac{2 H}{R_{T}}
$$

Tal linealización es válida, siempre y cuando los cambios en la altura y el flujo, a partir de sus valores respectivos en estado estable, sean pequeños. Otro concepto asociado a un sistema de fluido es la capacitancia C. Para el caso de un tanque, esta se define como el cambio necesario en la cantidad de líquido almacenado para producir un cambio de una unidad en el potencial (altura), esta es la cantidad que indica el nivel de energía del sistema. La capacitancia en el tanque se expresa mediante (9)

$$
C=\frac{\Delta v}{\Delta h},
$$

Donde $\Delta \mathrm{V}$ : cambio en el volumen del tanque $\left[\mathrm{m}^{3}\right]$ y $\Delta \mathrm{h}$ : cambio en la altura del nivel de líquido [m]. No deben ser confundidas la capacidad del tanque, expresada en $\left[\mathrm{m}^{3}\right]$ y su capacitancia $\left[\mathrm{m}^{2}\right]$. La capacitancia del tanque es igual a su área transversal, y si esta es constante, la capacitancia es constante para cualquier altura.

\subsubsection{Modelado y comportamiento de un sistema de control de nivel [3]}

La ecuación diferencial que rige la evolución temporal del sistema de nivel mostrado en la figura 2 se obtiene considerando que el flujo de entrada se ve disminuido en la cantidad del flujo de salida durante un pequeño intervalo de tiempo, y la disminución equivale a la cantidad adicional de líquido almacenada en el tanque. Lo expresado es equivalente a (10).

$$
C d h=\left(q_{i}-q_{0}\right) d t
$$

Donde $\bar{Q}$ : velocidad de flujo en estado estable antes de cualquier cambio; $\mathrm{q}_{\mathrm{i}}$ : desviación de la velocidad de entrada del flujo en estado estable; qo: desviación de la velocidad de salida del flujo en estado estable; $\bar{H}$ : altura de la columna de líquido en el tanque en estado estable; h: desviación de la altura a partir de su valor en estado estable; dh: diferencial de altura, y dt: pequeño tiempo transcurrido. A partir de la definición genérica de resistencia aplicada a flujo en este caso, la relación entre qo $\mathrm{y}$ h se expresa mediante (11)

$$
q_{0}=\frac{h}{R}
$$

Donde R: resistencia impuesta por la válvula a la descarga del tanque. La ecuación diferencial para este sistema, asumiendo un valor constante de R, luego de remplazar la ecuación (11) en la (10) se convierte en (12).

$$
R C \frac{d h}{d t}+h=R q_{i}
$$

Se observa de la ecuación (12) que la ecuación dinámica del sistema corresponde a una ecuación diferencial de primer orden, y que el término "RC" es la constante de tiempo del sistema. Tomando transformada de Laplace a ambos lados de la ecuación (12) y estableciendo como cero la condición inicial se obtiene (13)

$$
(R C s+1) H(s)=R Q_{i}(s)
$$

Donde las transformadas de las funciones $\mathrm{h}(\mathrm{t})$ y $\mathrm{q}_{\mathrm{i}}(\mathrm{t})$ están dadas por (14)

$$
H(s)=\mathcal{L}[h], \quad Q_{i}(s)=\mathcal{L}\left[q_{i}\right]
$$

Estableciendo la altura del líquido h como la variable de salida a controlar, y $\mathrm{q}_{\mathrm{i}}$ como el caudal o entrada al sistema, la función de transferencia del sistema es (15) 


$$
\frac{H(s)}{Q_{i}(s)}=\frac{R}{R C s+1}
$$

La entrada que genera el cambio de nivel en el tanque se modela como tipo escalón, dada por (16).

$$
q_{i}(t)=A u(t)
$$

Donde A: magnitud de la excitación y $\mathrm{u}(\mathrm{t})$ : función escalón unitario, véase figura 3(a), $\mathrm{t}_{0}$ es el tiempo inicial de excitación y la función escalón se muestra en la figura 3(b). La transformada de Laplace tomada a la ecuación (16) permite encontrar el modelado de la entrada en el dominio complejo dado por (17).

$$
Q_{i}(s)=\frac{A}{s}
$$

Que al ser remplazada en la ecuación (15) permite encontrar la salida del sistema o nivel del tanque en el dominio complejo mediante (18).

$$
H(s)=\frac{R A}{R C s+1}
$$

Aplicando transformada inversa de Laplace a la ecuación (18) con condición inicial $\mathrm{h}(0)=\mathrm{h}_{0}$, se obtiene (19).

$$
h(t)=R A\left(1-R A e^{-\frac{t}{R C}}\right)
$$

Donde $\tau$ : constante de tiempo del sistema dada por (20)

$$
\tau=R C \text {. }
$$

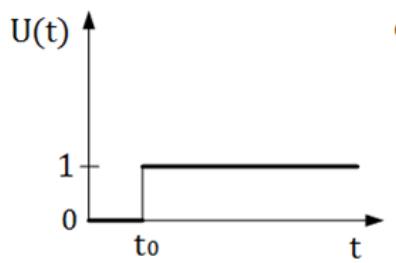

(a) Escalón unitario

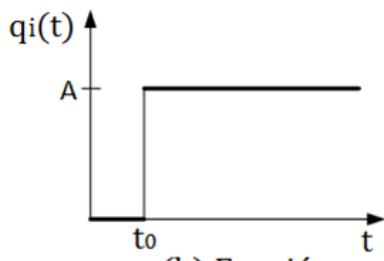

(b) Función escalón
Figura 3. Función escalón unitario y escalón. Fuente: elaboración propia.

La representación de la respuesta $\mathrm{h}(\mathrm{t})$ se ilustra en la figura 4, en función de la constante de tiempo del sistema, $\tau$. La respuesta $\mathrm{h}(\mathrm{t})$ ante una entrada tipo escalón alcanza el $63,2 \%$ de su respuesta en estado estable transcurrido un tiempo de $\tau$, y su límite de estado transitorio se alcanza al 99,3\% de la respuesta en un tiempo de $5 \tau$, considerado el límite entre estados transitorio y estable.

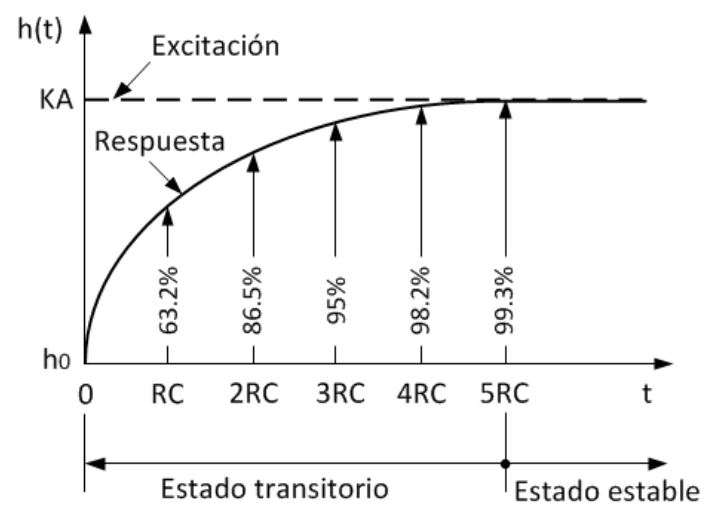

Figura 4. Respuesta del nivel del tanque h en función de la constante de tiempo. Fuente: elaboración propia.

\subsubsection{Modelado del sensor}

Existen tres términos básicos imprescindibles para establecer la operación de un instrumento de medición: la escala, el rango y el cero del instrumento. La escala del instrumento se especifica mediante un valor superior y otro inferior, mientras que el rango es la diferencia entre los valores. El cero del instrumento no necesariamente coincide con el cero de la variable medida. Un manómetro con rango de medida entre 10 psi y 70 psi tiene su cero de instrumento en 10 psi, mientras que el cero de la variable es de 0 psi.

El proceso de medición en un lazo de control se hace con sensores, en los cuales un fenómeno mecánico, eléctrico o similar es relacionado con la variable de proceso que se mide. En una etapa de transducción, se convierte la variable de proceso en una señal que se puede transmitir, llevando así una señal de proceso a un lazo de control. Un parámetro importante del sensor-transductor es la ganancia, que indica cuánto cambia la variable de salida, usualmente en $\mathrm{mA}$ o $\mathrm{mV}$, por unidad de cambio en la función de forzamiento o variable de entrada. También conocida como la ganancia del instrumento, la sensibilidad $\mathrm{K}$ del mismo se define mediante la razón del cambio en la variable de salida al cambio en la variable de entrada [3]:

$$
K=\frac{y_{f}-y_{i}}{x_{f}-x_{i}}
$$

Donde $\mathrm{y}_{\mathrm{f}}$ : valor final alcanzado por la señal de salida, $\mathrm{y}_{\mathrm{i}}$ : valor inicial de la señal de salida, $\mathrm{x}_{\mathrm{f}}$ : valor final alcanzado por el instrumento ante el cambio en el proceso y $\mathrm{x}_{\mathrm{i}}$ : lectura inicial en la variable del proceso antes de la transducción. La respuesta dinámica de la mayoría de los sensores-transductores es mucho (debe ser) más rápida que la del proceso, por lo cual sus constantes de tiempo y tiempo muerto se pueden considerar despreciables. Con esta consideración, la función de transferencia de un 
instrumento se considera la ganancia pura. Sin embargo, un enfoque más detallado al analizar la dinámica del instrumento es la consideración genérica de un sistema de primer o segundo orden [3].

\subsubsection{Modelado de la válvula de control}

Las válvulas de control son los elementos finales de control más usuales y se los encuentra en las plantas de proceso, en las cuales se manejan los flujos para mantener en los puntos de control las variables que se deben controlar [3]. La válvula de control actúa como una resistencia variable en la línea de proceso, mediante el cambio de su apertura se modifica la resistencia al flujo $\mathrm{y}$, consecuentemente, el flujo mismo, por lo que se consideran elementos reguladores de flujo. El dimensionamiento de la válvula de control es el procedimiento mediante el cual se calcula el coeficiente de flujo de la válvula, $\mathrm{C}_{\mathrm{v}}$. Este parámetro ha tenido una amplia aceptación entre los fabricantes de válvulas, propuesto por la empresa Masoneilan International, Inc., en 1944 [21]. El conocimiento del coeficiente $C_{v}$ de la válvula $\mathrm{y}$ del tipo de válvula requerido permite determinar el tamaño de la válvula con base en el catálogo de un fabricante. El coeficiente $C_{v}$ se define como «la cantidad de agua en galones U.S. que fluye por minuto a través de una válvula completamente abierta con una caída de presión de 1 psi en la sección transversal de la válvula» [22]. Por ejemplo; a través de una válvula con coeficiente máximo de 25 deben pasar 25 gpm de agua, cuando se abre completamente y la caída de presión es de 1 psi. La ecuación básica para dimensionar una válvula de control que se utiliza con líquidos es la misma para todos los fabricantes:

$$
q=C_{v} \sqrt{\frac{\Delta P}{G_{f}}}
$$

La cual se reescribe como (23).

$$
C_{v}=q \sqrt{\frac{G_{f}}{\Delta P}}
$$

Donde $q$ : flujo de líquido en gpm usando galones americanos, $\Delta \mathrm{P}$ : caída de presión en psi al paso por la misma, $\mathrm{G}_{\mathrm{f}}$ : gravedad específica del líquido a la temperatura en que fluye (entre $1^{\circ} \mathrm{F}$ a $60^{\circ} \mathrm{F}$ ).

\subsubsection{Función de transferencia del sistema de control de nivel}

La figura 5 presenta el sistema de control en lazo cerrado para controlar el sistema de nivel del tanque, con la ecuación del controlador tipo Proporcional, Integral y Derivativo (PID) está dada por la ecuación (24) [2], donde $K_{P}, K_{I}$ y $K_{D}$ son las ganancias proporcional, integral y derivativa del controlador, respectivamente. También de la figura 5, se distingue la ganancia del sensor K, la cual depende de la transducción del mismo.

$$
G(s)=K_{P}+\frac{K_{I}}{s}+K_{D} s
$$

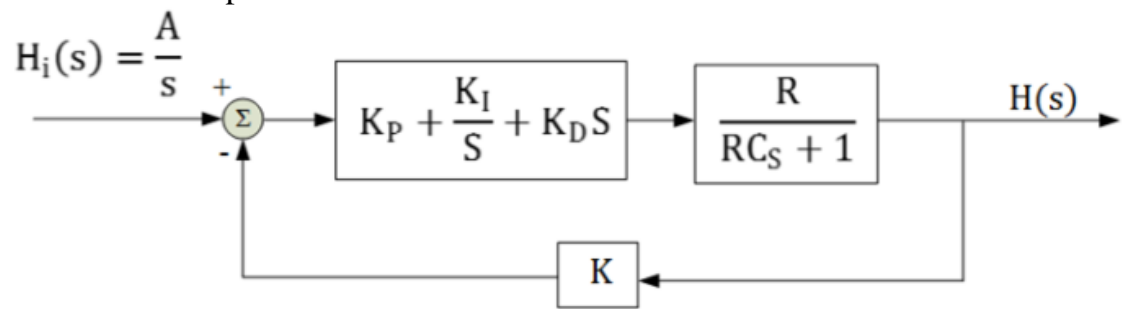

Figura 5. Sistema de control de nivel por retroalimentación para un tanque. Fuente: elaboración propia.

La Ecuación (24) se usa en el dominio temporal y se reescribe en función de una sola ganancia $\mathrm{K}_{\mathrm{P}}$, mediante la inclusión de los tiempos derivativos e integrales, también llamados rapidez derivativa e integral, denotadas por $\tau_{\mathrm{D}} \mathrm{y} \tau_{\mathrm{I}}$, respectivamente, para obtener (25).

$$
m(t)=\bar{m}+K_{P} e(t)+\frac{K_{P}}{\tau_{I}} \int e(t) d t+K_{P} \tau_{D} \frac{d e(t)}{d t},
$$

Donde m: salida del controlador (dominio del tiempo), $\bar{m}$ : consigna o valor de referencia, se han incluido las razones de cambio y derivada del error.

\subsection{Selección de componentes}

Se inicia estableciendo el volumen de agua que puede tener el sistema, 48 litros, de acuerdo con el rango de dimensiones de recipientes usados en bancos de laboratorio. Con este volumen se procede a determinar las especificaciones de la bomba, teniendo en cuenta que sea de $110 \mathrm{~V}$ de alimentación para facilitar su instalación en el laboratorio. 


\subsubsection{Selección de la bomba}

Los 48 litros de volumen de agua establecidos garantizan que el tanque no quedará vació, lo que evita que la bomba pierda el cebado. La presión de trabajo debe estar dentro del rango de la válvula o elemento de control, y no se requieren grandes presiones de trabajo, puesto que los circuitos típicos de bancos didácticos no incluyen pérdidas significativas. Consultando proveedores locales, se escogió una bomba centrífuga marca HYUNDAI, modelo HYJET80S, Potencia 0.75 HP, caudal máximo $50 \mathrm{lpm}$, con una cabeza de $45 \mathrm{~m}$, equivalente a 87 psi con una longitud de tubería de $10 \mathrm{~m}$ en tubería estándar de $1 / 2$ in en PVC. Las dimensiones esperadas del banco de laboratorio que determinan la longitud total de tubería, incluida la equivalente de los codos, uniones, válvulas de cierre, la misma válvula de control y demás, no excede este número.

\subsubsection{Selección del sensor}

Para el sensor de nivel se aborda inicialmente el siguiente análisis. Asumiendo un diámetro de $40 \mathrm{~cm}$ para el tanque de agua, los 10 litros de líquido se contienen en un tanque de al menos $80 \mathrm{~cm}$ de altura. Asumiendo $20 \mathrm{~cm}$ por encima del nivel de líquido para evitar derramamiento, el sensor de nivel debe tener un rango de lectura entre 10 $\mathrm{cm}$ y $100 \mathrm{~cm}$. De otra parte, para la selección de la válvula o actuador, se tiene en cuenta que soporte el caudal máximo de la bomba de $50 \mathrm{lpm}$, y que además soporte la presión generada por la bomba, de aproximadamente 87 psi. La válvula debe además estar integrada con los componentes de actuación y electrónicos que le permitan una acción de control combinando las acciones proporcional, integral y derivativo. Consultando proveedores locales, se determinó que el sensor con capacidad de transducción marca Flowline, modelo DL10 [23], rango de medida de 0 a $1 \mathrm{~m}$, y salida análoga de 4 a $20 \mathrm{~mA}$ se ajusta a las necesidades y cuya ganancia está dada por la ecuación (26). Para el sensor transmisor se considera que su constante de tiempo $\tau \rightarrow 0$, por lo cual la función de transferencia del sensor se aproxima a la ganancia $\mathrm{K}$ dada en la ecuación (26).

$$
K=\frac{(20-4) m A}{(100-0) c m}=0.192 \frac{m A}{C m}
$$

\subsubsection{Selección de la válvula}

Para la selección de la válvula de control se tienen en cuenta las características de la bomba, el tipo de circuito formado por la tubería y sus accesorios, la densidad del fluido y las necesidades mismas del proceso. La bomba tiene un caudal de $50 \mathrm{lpm}\left(3 \mathrm{~m}^{3} / \mathrm{h}\right)$, y una cabeza de $45 \mathrm{~m}$ de altura manométrica (63.76 psi). Para el cálculo de la constante de válvula, $\mathrm{K}_{\mathrm{V}}$, se seguirá lo dispuesto por A. Creus [24]. Se parte de un caudal conocido, por lo que este se corrige aumentando un $25 \%$, lo que arroja un caudal máximo de $3,75 \mathrm{~m}^{3} / \mathrm{h}$. Las pérdidas del circuito por fricción por accesorios se estiman en $6 \mathrm{~m}$, equivalente a 2 uniones universales, 4 codos, 5 válvulas de cierre rápido y una longitud de tubería de $2,5 \mathrm{~m}$ aproximadamente. En cuanto a las pérdidas por diferencia de nivel se estima en $1 \mathrm{~m}$. Con lo anterior, las pérdidas totales en el diseño se estiman en $7 \mathrm{~m}$, y quedan disponibles para la válvula de control $38 \mathrm{~m}$, equivalentes a 53,8 psi (3.71 bar) que deben ser absorbidos por la válvula de control, esta es la caída de presión, $\Delta \mathrm{P}$, que se va a vencer en la válvula. La constante de la válvula, $K_{V}$, se puede entonces calcular mediante (27).

$$
K_{V}=Q \sqrt{\frac{\delta}{\Delta P}}
$$

Donde el caudal $Q$ aumentado es de $3,75 \mathrm{~m}^{3} / \mathrm{h}$, la densidad del agua, $\delta$, a temperatura ambiente es de 1 $\mathrm{kg} / \mathrm{dm}^{3}$, y la caída de presión $\Delta \mathrm{P}$ es de 3,71 bar, para obtener una válvula dimensionada en 1,94. En el diseño del banco de pruebas de nivel y la selección de la válvula de control, se pueden presentar dos fenómenos que son inherentes a este tipo de sistemas, como lo son la vaporización y la cavitación, cuyos efectos son perjudiciales para los componentes del sistema e influyen en la capacidad de control, ya que limitan la capacidad de la válvula, a partir de un determinado caudal crítico, lo que limita el paso del líquido y, por ende, el caudal no aumenta. Estos fenómenos indican la necesidad de modificar el caudal crítico aparente, mediante un coeficiente de corrección que tiene en cuenta la capacidad de recuperación de presión de la válvula presentado ampliamente en la referencia [24]. Se evalúan las posibles configuraciones en las que podría trabajar la válvula de control, para seleccionar la que mejor se adapte a las condiciones físicas y económicas. Se calcula el número de Reynolds a la que estará expuesto el flujo en la tubería, con base en la expresión (28).

$$
R_{e}=\left(\frac{N_{4} F_{d} Q}{v \sqrt{K_{V} F_{L}}}\right) \sqrt[4]{\frac{F_{L}^{2} K_{V}^{2}}{N_{2} D^{4}}}+1
$$

Donde, $\mathrm{N}_{4}$ y $\mathrm{N}_{2}$ son constantes numéricas [24], $\mathrm{F}_{\mathrm{D}}$ : factor de modificación del tipo de válvula, $\mathrm{Q}$ : caudal, $\mathrm{v}$ : viscosidad cinemática $\left[\mathrm{m}^{2} / \mathrm{s}\right], \mathrm{K}_{\mathrm{V}}$ : coeficiente de válvula [ $\left.\mathrm{m}^{3} / \mathrm{h}\right], \mathrm{F}_{\mathrm{L}}$ : factor de recuperación de presión en líquidos y D: tamaño de la válvula. Los cálculos arrojan un número de Reynolds $\operatorname{Re}=142.000$, lo cual clasifica el flujo como turbulento. Se presentan varias configuraciones de funcionamiento, para cada una de las cuales se evalúa su condición de flujo mediante la 
verificación de las relaciones dadas en la ecuación (29) para flujo en condiciones subcríticas sin conos reductores, en la ecuación (30) para flujo en condiciones subcríticas con conos reductores, y en la ecuación (31) para flujo en condiciones críticas con y sin conos reductores, respectivamente:

$$
\begin{aligned}
& \Delta P_{c r}<F_{L}^{2}\left(P_{1}-\left(F_{F} P_{V}\right)\right), \\
& \Delta P_{c r}<\left(\frac{F_{L P}}{F_{P}}\right)^{2}\left(P_{1}-\left(F_{F} P_{V}\right)\right), \\
& \Delta P_{c r} \geq\left(\frac{F_{L P}}{F_{P}}\right)^{2}\left(P_{1}-\left(F_{F} P_{V}\right)\right),
\end{aligned}
$$

Donde $\Delta \mathrm{P}_{\mathrm{cr}}$ : presión crítica; $\mathrm{F}_{\mathrm{LP}}$ : factor de recuperación del líquido combinado con el factor de geometría de la tubería de la válvula de control con conos reductores; $\mathrm{F}_{\mathrm{P}}$ : factor de geometría de la tubería de la válvula de control; $\mathrm{P}_{1}$ : presión en la entrada de la válvula; $\mathrm{F}_{\mathrm{F}}$ : factor de recuperación de presión en líquidos sin conos reductores, y $\mathrm{P}_{\mathrm{v}}$ : presión de vapor del líquido. Para el caso considerado se tienen los valores de $\mathrm{F}_{\mathrm{L}}=0,90, \mathrm{~F}_{\mathrm{LP}}=0,90$, $\mathrm{F}_{\mathrm{P}}=0,99, \mathrm{~F}_{\mathrm{F}}=0,95, \mathrm{P}_{\mathrm{V}}=0,04$ bar, y $\mathrm{P}_{1}=4,22$ bar. Al ser reemplazados en las ecuaciones (29) y (30) se verifica el cumplimiento de la condición de la ecuación 31 , por lo cual se tienen condiciones críticas (3.71 bar $\geq 3.37$ bar). El nuevo valor del coeficiente de la válvula para condiciones críticas viene dado por (32).

$$
C=\frac{Q}{N_{1} F_{L}} \sqrt{\frac{\rho}{P_{1}-F_{F} P_{V}}}
$$

Donde $\mathrm{N}_{1}=1$ es una constante numérica de aplicación a las fórmulas del coeficiente de válvula [20], [24] y el resto de variables ya han sido definidas anteriormente. Reemplazando los valores en la ecuación 32 se tienen los valores de los nuevos $K_{v}$ en la tabla 1, para cada una de las diferentes configuraciones. El fabricante de la válvula reporta un $\mathrm{K}_{\mathrm{v}}$ de 2,1 [25], con característica inherente lineal, es decir, a iguales incrementos de la carrera iguales incrementos en el caudal, y las características de caudal efectivas vienen dadas por (33).

$$
q_{e}=\frac{1}{\sqrt{1-r+\frac{r}{q_{i}^{2}}}}
$$

Donde $\mathrm{q}_{\mathrm{i}}$ : característica de caudal inherente dada por la ecuación (34), r: relación entre la pérdida de carga que absorbe la válvula $\mathrm{H}_{1}$ y la pérdida total del sistema $\mathrm{H}$ [24], expresado por la ecuación (35).

$$
q_{i}=K l
$$

$$
r=\frac{H_{1}}{H}
$$

Tabla 1. Valores de Kv con características de caudal efectivo e inherente, con y sin conos reductores.

\begin{tabular}{|l|c|c|c|c|}
\hline \multicolumn{1}{|c|}{ Caudal } & $\mathbf{r}$ & $\mathbf{K}_{\mathbf{v}}$ & $\begin{array}{c}\mathbf{K}_{\mathbf{v}} \text { Sin } \\
\text { Conos }\end{array}$ & $\begin{array}{c}\mathbf{K}_{\mathbf{v}} \text { Con } \\
\text { Conos }\end{array}$ \\
\hline \multirow{2}{*}{ Nominal } & 0,911 & 1,51 & 1.628 & \\
\cline { 2 - 5 } & 0,988 & 1,45 & & 1.604 \\
\hline \multirow{2}{*}{ Aumentado } & 0,862 & 1,94 & 2.056 & \\
\cline { 2 - 5 } & 0,983 & 1,82 & & 2.001 \\
\hline
\end{tabular}

Fuente: elaboración propia, adaptada de [22].

La figura 6 presenta las características lineal y efectiva de la válvula, para un sistema que trabaja con el caudal nominal de la bomba y con una configuración de la tubería con conos y sin conos reductores, y para otro sistema que trabaja con un aumento en el caudal nominal de la bomba del $25 \%$, con una configuración de la tubería con conos y sin conos reductores.

La tabla 1 también presenta los diferentes valores que toman $\mathrm{K}_{\mathrm{V}}$ y $\mathrm{r}$ para cada una de las posibles configuraciones del sistema. A partir de la Figura 6 y la tabla 1, se procede a seleccionar la válvula de control para el circuito de nivel. Se escoge una que pueda satisfacer cualquiera de las cuatro configuraciones posibles que se tienen, con un circuito de tuberías de diámetro nominal de 0,5 in, y el caudal nominal de la bomba. Se obtiene un valor de $\mathrm{r}=0.911$, y un $\mathrm{K}_{\mathrm{V}}=1.51$ bajo condiciones normales de operación, y $\mathrm{K}_{\mathrm{V}}=1.628$ bajo condiciones críticas. El valor de $\mathrm{K}_{\mathrm{v}}=1.628$ es el que se comparará contra el coeficiente de válvula ofrecido por el fabricante. La válvula seleccionada es electroválvula proporcional servoaccionada de 2 vías modelo EV260B 15B [25], con valor de $\mathrm{K}_{\mathrm{V}}=2.1\left[\mathrm{~m}^{3} / \mathrm{h}\right]$, como valor más cercano y por encima al obtenido mediante cálculos. El dimensionamiento de la válvula de control también puede hacerse de manera general con el catálogo del fabricante, teniendo en cuenta la ecuación (36), definida para agua (densidad relativa de uno) a $20^{\circ} \mathrm{C}$, con una pérdida de presión $\Delta \mathrm{P}$ en bares, y el caudal q en $\mathrm{m}^{3} / \mathrm{h}$.

$$
K_{V}=q \sqrt{\frac{1}{\Delta P}}
$$

Para un caudal nominal de la bomba de $3 \mathrm{~m}^{3} / \mathrm{h}$ y el diagrama de capacidad de la válvula para agua completamente abierta se lee del catálogo [25] un diferencial de presión equivalente a 2,1 bar. Al ser sustituidos estos valores en la ecuación (36) se obtiene un $\mathrm{K}_{\mathrm{V}}=2.068$ que concuerda con $\mathrm{K}_{\mathrm{v}}=2.1$ del catálogo. 


\subsubsection{Selección de la válvula}

Para el proceso se tiene una válvula de característica lineal en todo el rango, entre la posición de la válvula y la salida del controlador, con caída de presión constante, mediante la linealización de la ecuación (37), se obtiene la ganancia de la válvula igual a $0.003125\left(\mathrm{~m}^{3} / \mathrm{min} \times\right.$ $\mathrm{mA}$ ). La constante de tiempo para la válvula de control tiende a $0, \tau \rightarrow 0$, por lo cual la función de transferencia de la válvula de control es igual a la ganancia de la válvula.

$$
Q=Q_{\max } V_{P}=Q_{\max } \frac{m-4}{20-4}
$$

\subsection{Dinámica del sistema}

Para el proceso real de la figura 7 se tiene interés en conocer cómo responde el nivel $\mathrm{h}(\mathrm{t})$ del líquido en el tanque a los cambios de flujo de entrada $\mathrm{q}_{\mathrm{i}}(\mathrm{t})$, y a los cambios en la apertura de la válvula de salida, $\mathrm{V}_{\mathrm{P}}(\mathrm{t})$. El caudal de salida de un tanque con descarga a la atmósfera viene representado por la ecuación (38), donde $\mathrm{q}_{\mathrm{i}}(\mathrm{t})$ : flujo de entrada $\left[\mathrm{m}^{3} / \mathrm{h}\right], \mathrm{q}_{\mathrm{o}}(\mathrm{t})$ : flujo de salida $\left[\mathrm{m}^{3} / \mathrm{h}\right], \mathrm{C}_{\mathrm{d}}$ : coeficiente adimensional de descarga, $\mathrm{V}_{\mathrm{P}}$ : posición de la válvula que representa su fracción de apertura $(0$, indica que la válvula está cerrada, y 1 indica que está completamente abierta), $\mathrm{A}_{\mathrm{T}}$ : área transversal de la tubería $\left[\mathrm{m}^{2}\right]$, g: gravedad en $\mathrm{m} / \mathrm{s}^{2}, \Delta \mathrm{h}$ : diferencia de altura $[\mathrm{m}]$.

$$
q(t)=C_{d}(v p(t)) A_{t} \sqrt{2 g \Delta h}
$$

Del balance de masa en estado estacionario al sistema de la figura 7 se obtiene la ecuación (39), donde A representa el área transversal del tanque en $\mathrm{m}^{2}$. La densidad en el sistema es igual en la entrada y la salida por lo cual se obtiene la ecuación (40).

A continuación se definen las variables de desviación en términos de los términos linealizados, y se sustituyen en la ecuación diferencial, para obtener:

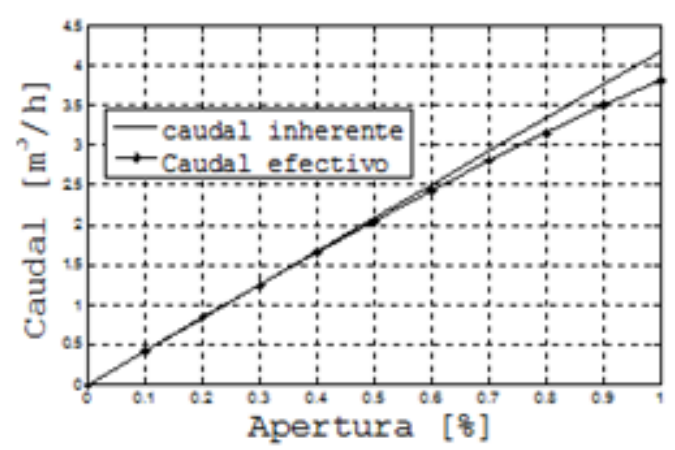

a) Caudal nominal con conos reductores

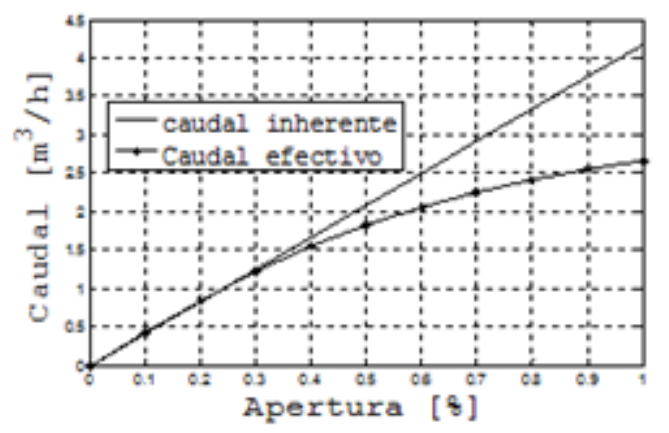

b) Caudal nominal sin conos reductores

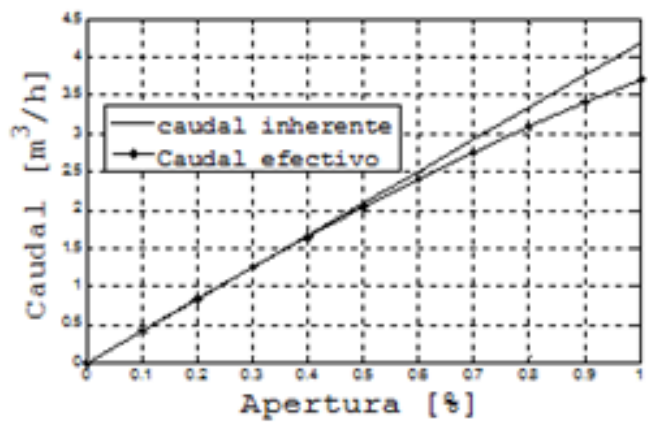

c) Caudal aumentado con conos reductores

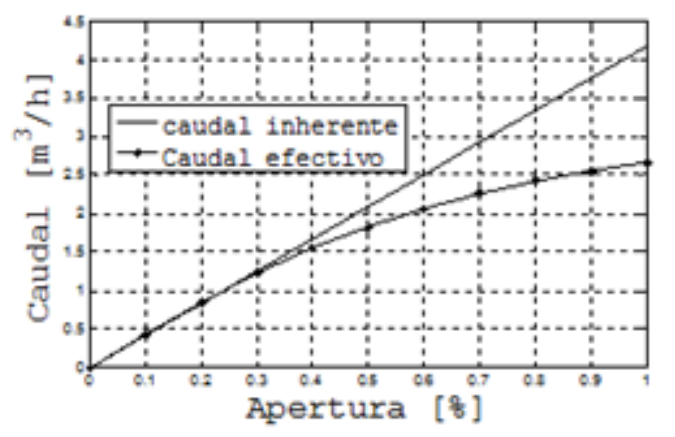

d) Caudal aumentado sin conos reductores

Figura 6. Caracterísiticas de caudal inherente y efectivo, con y sin conos. Fuente: elaboración propia. 


$$
\begin{gathered}
Q_{i}(t)=q_{i}(t)-\bar{q}_{i} \\
V P(t)=v p(t)-\overline{v p} \\
H(t)=h(t)-\bar{h} \\
Q_{i}(t)-C_{1} V P(t)-C_{2} H(t)=A \frac{d H(t)}{d t},
\end{gathered}
$$

Se reordena la ecuación (42):

$$
\tau \frac{d H(t)}{d t}+H(t)=K_{1} Q_{i}(t)-K_{2} V P(t)
$$

Donde,

$$
\begin{aligned}
& \tau=\frac{A}{C_{2}} \\
& K_{1}=\frac{1}{C_{2}} \\
& K_{2}=\frac{C_{1}}{C_{2}} .
\end{aligned}
$$

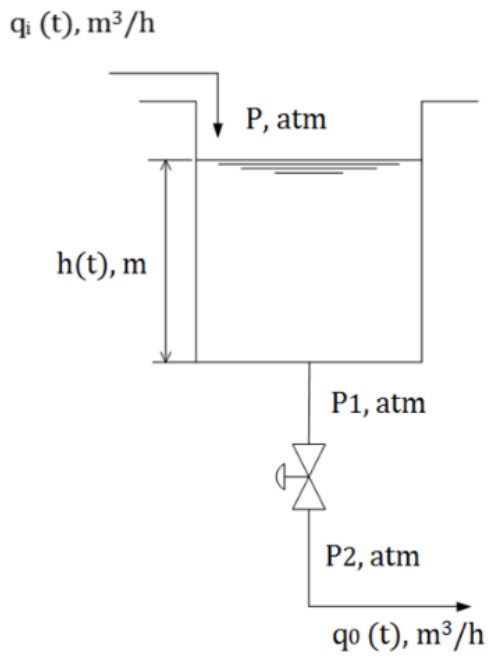

Figura 7. Sistema del proceso del nivel. Fuente: elaboración propia.

Finalmente, se obtiene la transformada de Laplace, ecuación (47) que representa la función de transferencia del proceso

$$
H(s)=\frac{K_{1}}{\tau s+1} Q_{i}(s)-\frac{K_{2}}{\tau s+1} V P(s),
$$

A partir de la cual se determinan las funciones de transferencia del proceso:

$$
\frac{H(s)}{Q_{i}(s)}=\frac{K_{1}}{\tau s+1}
$$

$$
\frac{H(s)}{V P(s)}=\frac{K_{2}}{\tau s+1} .
$$

\subsubsection{Elementos y dimensionamiento del sistema}

El conjunto de válvula y sensor escogido facilita el manejo de señales y la acción de control, dado que las señales de ambos elementos son análogas, y no se necesita convertidor análogo-digital. La compatibilidad de estos elementos con el PLC Siemens S7300 de 4 entradas y 2 salidas análogas, combinado con su capacidad de acción de control proporcional, integral y derivativo, completa la selección de los elementos. Además, el PLC permite las operaciones entre las señales para generar la señal de error. La Figura 8 resume las características de los elementos escogidos.

El circuito de proceso debe incluir válvulas $\mathrm{y}$ componentes que permitan la manipulación del sistema, tales como codos, uniones, válvulas manuales de globo, tanque de almacenamiento de líquido, manómetro, entre otros. En la Figura 9 se presenta un diseño preliminar del circuito de proceso con la respectiva nomenclatura de Diagrama de Proceso e Instrumentación (P\&ID). El nivel a controlar es del volumen de agua contenido dentro del tanque TK nivel, cuyo fluido es tomado de un depósito mediante una bomba centrífuga. Se muestra también la ubicación del sensor de nivel con capacidad de transducción; la ubicación de la válvula de control proporcional impulsada, número 1; la válvula manual reguladora de flujo (2), y las válvulas manuales de globo para generar perturbaciones en el sistema.

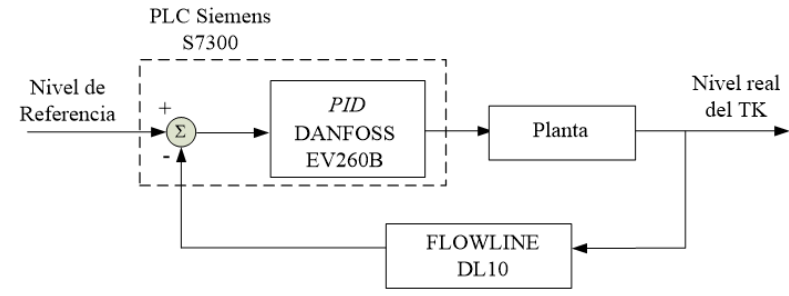

Figura 8. Esquema de hardware seleccionado. Fuente: elaboración propia.

La figura 10 presenta el banco del esquema P\&ID de la figura 9. Se identifican los componentes: 1-Transmisor ultrasónico de nivel, 2-Pantalla de visualización LCD, 3Válvula de control proporcional, 4-Tablero eléctrico de control, 5-Tablero de conexiones, 6-Bomba centrifuga, 7-Tanque de almacenamiento, 8-Tanque de proceso, 9Válvula de compuerta, 10-Válvula de globo. Los datos físicos del proceso del banco en la figura 9 son: $A=3.75 \times 10^{-2} \mathrm{~m}^{2}, A_{t}=1.32 \times 10^{-4} \mathrm{~m}^{2}, \bar{h}=0.40 \mathrm{~m}$, $g=9.81 \mathrm{~m} / \mathrm{s}^{2}, \quad \overline{v p}=0.3, \quad \rho=3800 \mathrm{~kg} / \mathrm{m}^{3}, \quad Q_{i}=$ 
$0.72 \mathrm{~m}^{3} / \mathrm{h}, Q_{o}=0.72 \mathrm{~m}^{3} / \mathrm{h}, C_{d}=0.332$, los cuales permiten calcular $C_{1}=1.22 \times 10^{-4} \mathrm{~m}^{3} / \mathrm{s}, C_{2}=5.34 \times$ $10^{-5} \mathrm{~m}^{2} / \mathrm{s}$ y los valores de los coeficientes $\tau=$ $11.7 \mathrm{~min}, \quad K_{1}=312 \mathrm{~min} / \mathrm{m}^{2} \quad$ y $\quad K_{2}=2.29 \mathrm{~m}$, reemplazados en la ecuación (49) y obtener la función de transferencia en la ecuación (50).

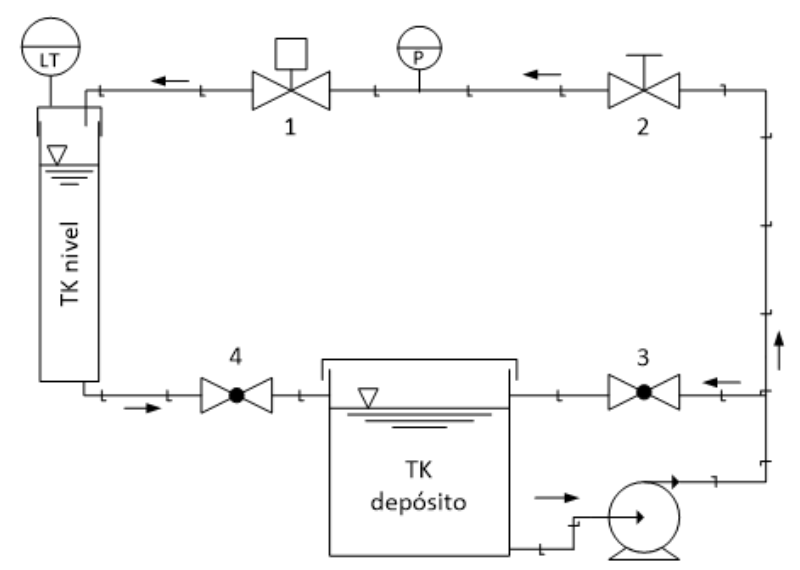

Figura 9. Diagrama de proceso (P\&ID) de control de nivel en el tanque. Fuente: elaboración propia.

$$
H(s)=\frac{312}{11.7 s+1} Q_{i}(s)-\frac{2.29}{11.7 s+1} V P(s)
$$

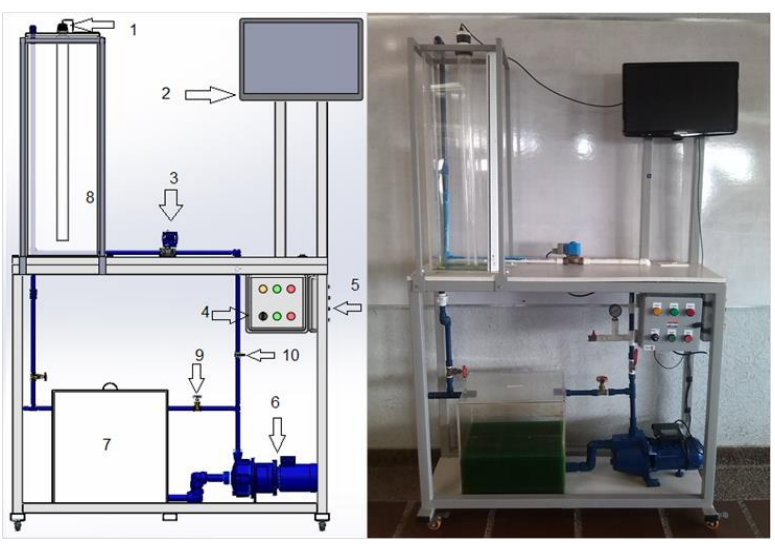

Figura 10. Banco construido para implementación del control de nivel. Fuente: elaboración propia.

\subsubsection{Función de transferencia del sistema}

La figura 11 presenta el diagrama de bloques del circuito del banco en la figura 10 , con $\mathrm{M}(\mathrm{S})$ como la salida del controlador, y la señal del trasmisor es $\mathrm{C}(\mathrm{s})$, lo que genera la función $\mathrm{G}(\mathrm{s})$ dada por la ecuación (50). Tomando transfomada de Laplace a la ecuación (25) y reemplazando valores del proceso en las funciones de transferencia de las ecuaciones (49) y (48); con las funciones transferencia para la válvula de control y el sensor-transmisor determinadas como $\mathrm{G}_{\mathrm{V}}(\mathrm{S})=0.003125 \mathrm{y}$ $\mathrm{G}_{\mathrm{T}}(\mathrm{S})=19.2$, respectivamente, se obtiene la ecuación característica del proceso dada en la ecuación (52).

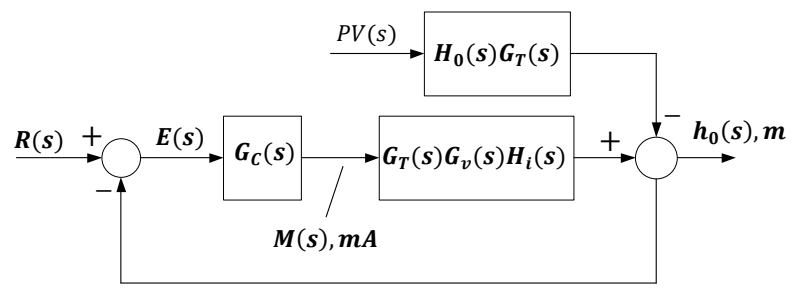

Figura 11. Diagrama de bloques del banco de control de nivel. Fuente: elaboración propia.

A partir de la ecuación (52) se genera el polinomio característico del proceso, dado en la ecuación (53). Los parámetros de ajuste del controlador $K_{P}, \tau_{D}$ y $\tau_{i}$ serán hallados por medio de métodos experimentales.

$$
\begin{gathered}
G(s)=G_{T}(s) G_{V}(s) H_{i}(s) \\
1+H_{i}(s) G_{v}(s) G_{d}(s) G_{t}(s)=0 \\
s^{2}\left(\tau_{i}+18.72 K_{P} \tau_{D} \tau_{i}\right)+s\left(0.085 \tau_{i}+18.72 K_{P} \tau_{i}\right)+ \\
18.72 K_{P}=0
\end{gathered}
$$

\subsection{Interfaz de usuario}

Para el diseño de la interfaz del PLC se tienen en cuenta los factores de desarrollo, de visibilidad y de aceptación [26], [27]. Los primeros establecen criterios para la mejora de la comunicación visual, los de visibilidad tienen en cuenta los factores humanos y expresan una fuerte identidad visual, y los factores de aceptación incluyen alguna plantilla preexistente. El desarrollo de la pantalla de visualización fue considerado para un ambiente industrial en plataforma SIMATIC WinCC flexible, de SIEMENS, la familia del PLC S7-300. Se tomó como como referencia la metodología propuesta para la guía ergonómica de diseño de interfaces de supervisión (GEDIS) enfocada a ambientes industriales con salas de supervisión computarizadas y centralizadas [28], en donde se ordena de lo general a lo particular.

Los aspectos GEDIS considerados se describen a continuación: Arquitectura: 4 pantallas de proceso; Distribución de las pantallas: Información del proceso de nivel y visualización de variables (set point, nivel real, y porcentaje de salida de la válvula); Navegación: Intuitiva a través de las 4 pantallas mediante botones indicadores; Uso del color: Fondo gris agradable a la vista y visualización de campos mediante contraste; Información textual: Información al usuario tanto en forma de texto como mediante gráficos debidamente 
rotulados y explicados; Estatus de los equipos y eventos: Se identifica si el proceso se encuentra activo $(O n)$ o apagado (Off) mediante colores, luces intermitentes e indicadores de texto; Información y valores de proceso: Es posible visualizar el cambio de nivel en tiempo real, el estado de la válvula y del sensor; Gráficos de tendencia y tablas: Ambos se usan como los principales medios de agrupamiento de las variables, se permite hacer un acercamiento (zoom) para detallar los valores; Comandos e ingreso de datos: Se permite asignar valores al set point y los valores de ganancias; Alarmas: un campo intermitente de color rojo indica la desconexión del banco con el PLC. La figura 12 muestra las pantallas desarrolladas, se distingue la pantalla principal, donde se establece la interconexión de los diferentes componentes del proceso, figura 12.a, y se da un acceso hacia las otras pantallas; véase figura 12.b. Se muestra la pantalla de control manual, donde se puede encender la bomba y regular la apertura de la válvula, figura 12.c, que también muestra gráficas en tiempo real del proceso y permite el control y visualización de los parámetros.

\subsection{Caracterización del proceso}

Para llevar a cabo el ajuste del controlador se caracteriza el proceso mediante la aproximación de la función de la ecuación (51) a un modelo simple de primer orden con tiempo muerto presentado en la ecuación (54), compuesto de la ganancia $\mathrm{K}$, el tiempo muerto $\mathrm{t}_{0}$, y la constante de tiempo $\tau$. Así, el problema se plantea como la determinación de estos parámetros para un proceso característico, y su solución consiste en realizar algunas pruebas dinámicas al circuito real, o la simulación por computadora, la prueba más sencilla que se puede realizar, es la entrada tipo escalón.

$$
G(S)=\frac{K e^{-t_{0} S}}{\tau s+1}
$$

\subsubsection{Conexión al PLC}

La conexión entre el banco y la bornera del PLC se realiza como se indica en la figura 13. La comunicación de señales se da a través de las señales $+\mathrm{A} 1$ y $+\mathrm{A} 0$.

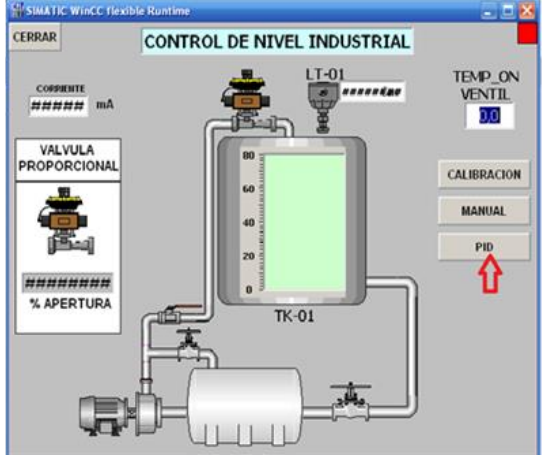

a) Pantalla principal

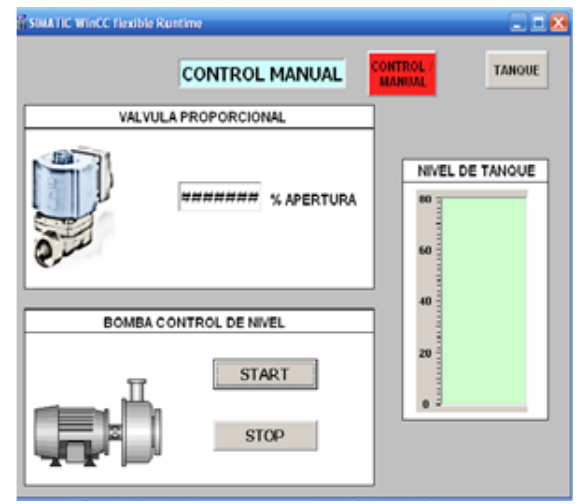

b) Pantalla control manual

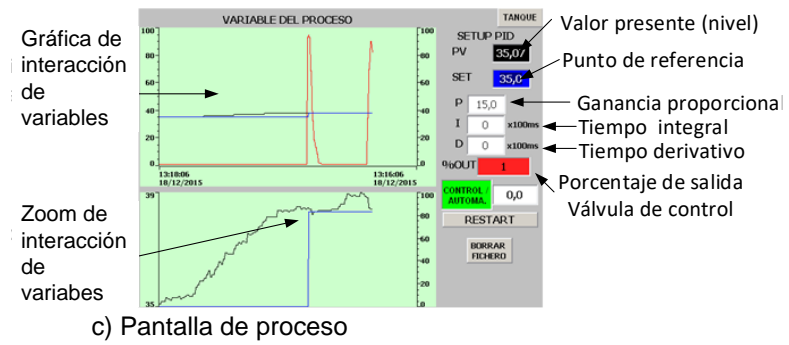

Figura 12. Pantallas principales de la interfaz para control de nivel. Fuente: elaboración propia.

\subsubsection{Mecánica de la prueba escalón}

El procedimiento de la prueba de escalón se lleva a cabo como sigue: 1) con el controlador en la posición "manual" (es decir, el circuito abierto), se aplica al proceso un cambio escalón en la señal de salida del controlador $\mathrm{M}(\mathrm{t})$; la magnitud del cambio debe ser lo suficientemente grande como para que se pueda medir el cambio consecuente en la señal de salida del transmisor, pero no tanto como para evitar que no linealidades del proceso distorsionen la respuesta. 2) La respuesta de la señal de salida del transmisor $\mathrm{C}(\mathrm{t})$ se grafica una escala adecuada tanto de amplitud como en la de tiempo, por al menos el período completo de la prueba, desde el inicio hasta alcanzar un nuevo estado estacionario. 3) Finalmente se hace coincidir la curva de reacción del 
proceso con el modelo de un proceso simple para determinar los parámetros del modelo.

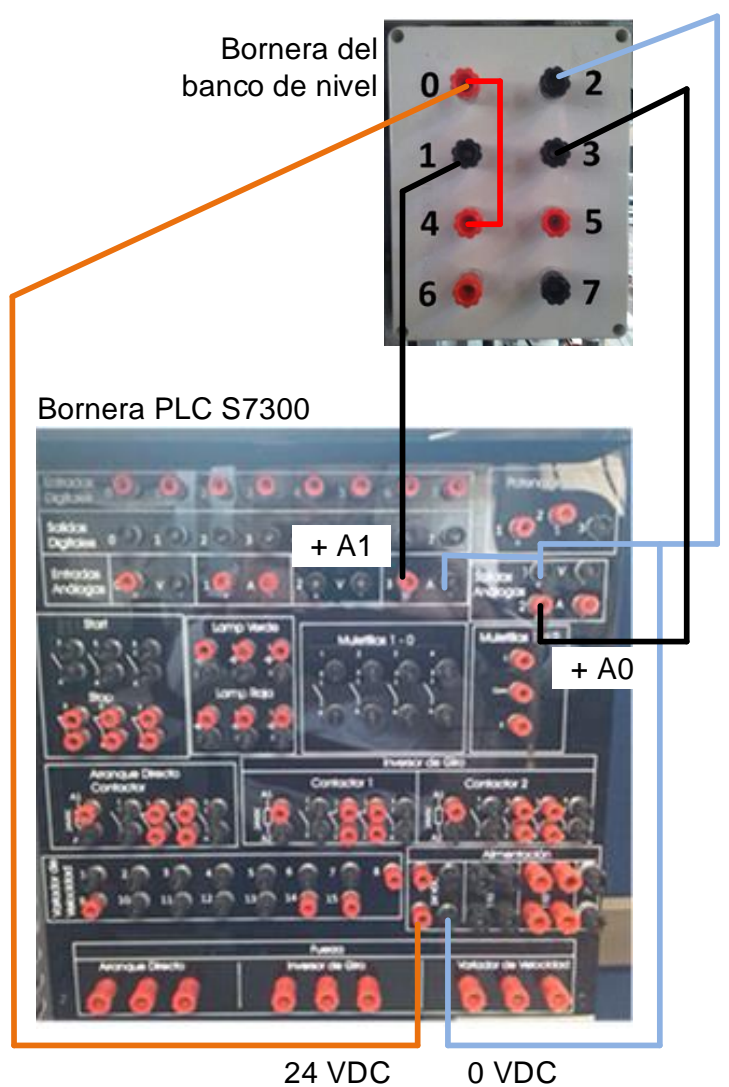

Figura 13. Conexiones y cableado entre banco y PLC. Fuente: elaboración propia.

La respuesta de la señal de salida del transmisor se expresa mediante la ecuación (55).

$$
C(S)=G(S) M(S)
$$

Para un cambio escalón de magnitud $\Delta \mathrm{m}$, en la salida del controlador y un modelo de primer orden más tiempo muerto se tiene (56).

$$
C(S)=\frac{K e^{-t_{0} S}}{\tau s+1}\left(\frac{\Delta m}{s}\right)
$$

La cual se expande en fracciones parciales y resuelta mediante transformada inversa de Laplace, se obtiene:

$$
\Delta c(t)=K \Delta m u\left(t-t_{0}\right)\left[1-e^{\frac{-\left(t-t_{0}\right)}{\tau}}\right]
$$

Donde $\mathrm{u}(\mathrm{t}-\mathrm{t} 0)$ representa la función escalón unitario, y se introduce para indicar:

$$
\Delta c(t)=0, \text { para } t \leq t_{0}
$$

$\mathrm{Y}$ para un tiempo mayor a $\mathrm{t}_{0}, \Delta_{\mathrm{c}}(\mathrm{t})$ representa la perturbación o cambio de salida del transmisor respecto a su valor inicial y dada por la ecuación (59), cuya salida se muestra en la figura 14 , donde el término $\Delta_{\mathrm{cs}}$, es el cambio en estado estacionario de $\Delta_{\mathrm{ct}}$.

$$
\Delta c(t)=c(t)-C(0)
$$

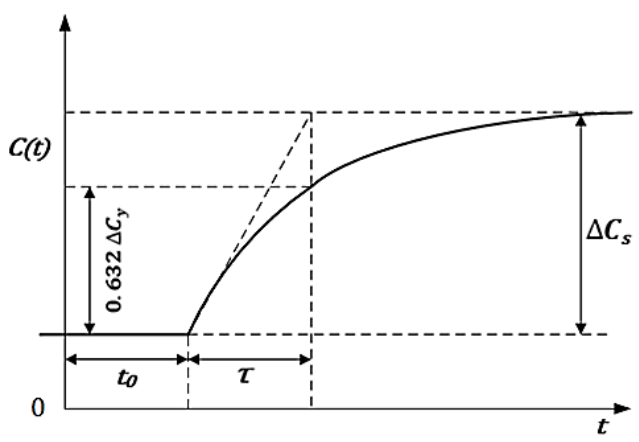

Figura 14. Respuesta escalón de un proceso de primer orden. Fuente: elaboración propia.

De la ecuación (59) es posible establecer que

$$
\Delta C_{s}=\lim _{t \rightarrow \infty} \Delta c(t)=K \Delta m,
$$

y a partir de esta, teniendo en cuenta que la respuesta del modelo debe coincidir con la curva de reacción del proceso en estado estable, se puede calcular la ganancia de estado estacionario del proceso mediante la ecuación (61). Este es uno de los parámetros del modelo, el tiempo muerto $\mathrm{t}_{0} \mathrm{y}$ la constante de tiempo $\tau$ se pueden determinar al menos mediante tres métodos, cada uno de los cuales genera diferentes valores.

$$
K=\frac{\Delta C_{S}}{\Delta m}
$$

\subsubsection{Prueba escalón al proceso de nivel del banco}

Los datos de la tabla 2 resumen las lecturas obtenidas al aplicar la prueba escalón, donde $\mathrm{h}_{\mathrm{R}}$ : nivel de referencia $\mathrm{o}$ set point, y h: altura real de nivel en el tanque. Esta tabla fue generada de lecturas en tiempo real arrojadas por el sensor ultrasónico y del temporizador del PLC, almacenadas inicialmente en formato ".csv", exportándolo a Excel. La lectura y registro de los datos del proceso de nivel puede verse afectado por diversos procesos internos que corren de manera simultánea en el PLC, pero en ningún caso será por más de un segundo consecutivo. Los ciclos exclusivos para la lectura y escritura de los valores de $\mathrm{h}, \mathrm{h}_{\mathrm{r}} \mathrm{y}$ el porcentaje de salida, varían entre 52 y 58 segundos, por lo cual se considera 
que esta característica de la lectura de datos no afectará las mediciones del sistema, ya que se realizan mediciones de nivel en cm, y es físicamente imposible para el banco de pruebas, cambiar $1 \mathrm{~cm}$ de nivel en 1 segundo.

Tabla 2. Comportamiento del sistema en lazo abierto.

\begin{tabular}{|c|c|c|}
\hline $\mathbf{t}[\mathbf{s}]$ & $\mathbf{h}_{\mathbf{R}}[\mathbf{c m}]$ & $\mathbf{h}[\mathbf{c m}]$ \\
\hline 0 & 40 & 0 \\
\hline 10 & 40 & 3 \\
\hline 20 & 40 & 7 \\
\hline 30 & 40 & 10 \\
\hline 40 & 40 & 14 \\
\hline 50 & 40 & 17 \\
\hline 60 & 40 & 19 \\
\hline 70 & 40 & 23 \\
\hline 80 & 40 & 26 \\
\hline 90 & 40 & 31 \\
\hline 100 & 40 & 34 \\
\hline 110 & 40 & 38 \\
\hline 120 & 40 & 40 \\
\hline
\end{tabular}

Fuente: elaboración propia.

La figura 15 muestra la dinámica del sistema en lazo abierto para los datos de la tabla 2. A partir de esta gráfica se toman los dos puntos descritos en el "método 3" sugerido por Smith and Corripio [3], donde los puntos 1 y 2, denotados por P1 y P2, respectivamente, están dados por las ecuaciones (62) y (63), respectivamente, y representan el $63 \%$ y el $28 \%$ del cambio de la variable controlada, como se presenta en las ecuaciones (64) y (65). La dinámica del sistema en lazo abierto se muestra en la figura 15.

$$
\begin{aligned}
& P_{1}=t_{0}+\frac{\tau}{3} \\
& P_{2}=t_{0}+\tau \\
& \Delta c]_{t_{0}+\tau}=0.632 \Delta c_{S} \\
& \Delta c]_{t_{0}+\tau / 3}=0.283 \Delta c_{S}
\end{aligned}
$$

Los valores de $t_{0} \mathrm{y} \tau$ se pueden obtener de la resolución de sistema de ecuaciones formado por las ecuaciones (66) y (67), para obtener los valores en las ecuaciones (68) y (69), donde $t_{1}$ es el tiempo en el cual $\Delta_{C}=0.283 \Delta_{\mathrm{CS}}$ y t $t_{2}$ el tiempo en el cual $\Delta_{\mathrm{C}}=0.632 \Delta \mathrm{C}_{\mathrm{S}}$.

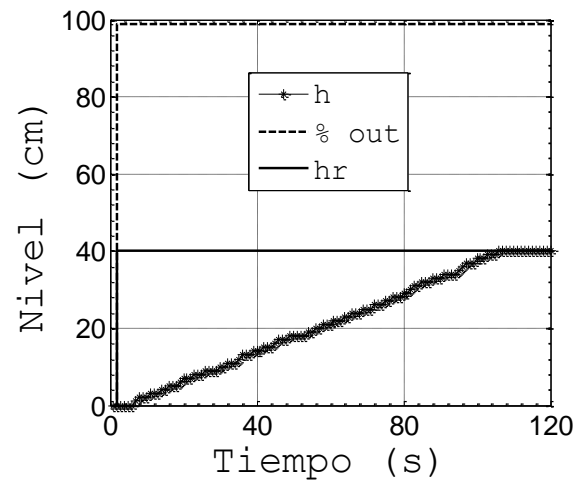

Figura 15. Respuesta del sistema en la prueba escalón. Fuente: elaboración propia.

$$
\begin{aligned}
& t_{0}+\tau=t_{2} \\
& t_{0}+\frac{\tau}{3}=t_{1} \\
& t_{0}=t_{2}-\tau \\
& \tau=\frac{3}{2}\left(t_{2}-t_{1}\right)
\end{aligned}
$$

Conociendo el valor de $\Delta \mathrm{C}_{\mathrm{S}}\left(\Delta \mathrm{C}_{\mathrm{S}}=40 \mathrm{~cm}\right)$, es posible hallar $\Delta \mathrm{C}$ para $28 \%$ y $63 \%$ de la respuesta mediante las ecuaciones (68) y (69), de lo que se obtiene para $28 \%$ $(\Delta \mathrm{C} 1=0,283 \mathrm{~cm})$ un $\Delta \mathrm{C}=11,32 \mathrm{~cm}$, y para el $63 \%$ $(\Delta \mathrm{C} 2=0,632 \mathrm{~cm})$ un $\Delta \mathrm{C}=25,28 \mathrm{~cm}$. A continuación, se identifican los tiempos en los cuales se alcanzan estos niveles $\Delta \mathrm{C}$, siendo necesaria la interpolación, a partir de los valores de la tabla 3 , para obtener $\mathrm{t}_{1}=33,64 \mathrm{~s}$ y $\mathrm{t}_{2}=78,28 \mathrm{~s}$, para $28 \%$ y $63 \%$ de respuesta, respectivamente. Reemplazando los valores obtenidos de $\mathrm{t}_{1}$ y $\mathrm{t}_{2}$ en la ecuación (69) se halla $\tau=67,96 \mathrm{~s}$. A continuación, se reemplazan los valores de $\tau$ y $t_{2}$ en la ecuación (68) para obtener $t_{0}=11,31 \mathrm{~s}$. La ganancia de estado estacionario representada por la ecuación (61) es calculada a partir de $\Delta \mathrm{C}_{S}=40 \mathrm{~cm}$ y $\Delta \mathrm{m}=100 \mathrm{~cm}$, para un valor de $\mathrm{K}=0,40$. Reemplazando valores numéricos en la ecuación (57) se obtiene la expresión de la respuesta del sistema ante una entrada tipo escalón, presentada en la ecuación (70).

$$
\Delta c(t)=0.40 \Delta m u(t-11.31)\left[1-e^{-\frac{t-11.31}{66.96}}\right]
$$

El conocimiento de la dinámica del sistema $\left(K, \mathrm{t}_{0}\right.$ y $\left.\tau\right)$ permite determinar los parámetros del modelo, de acuerdo con el tipo de controlador, a partir de la tabla 4, de lo que se obtiene $K_{\mathrm{P}}=15$, para un controlador proporcional; $\mathrm{K}_{\mathrm{P}}=13.6$ y $\tau_{\mathrm{i}}=37 \mathrm{~s}$, para un controlador proporcional-integral; y $\mathrm{K}_{\mathrm{P}}=18.1, \tau_{\mathrm{i}}=22.3 \mathrm{~s}$ y $\tau_{\mathrm{D}}=5.54 \mathrm{~s}^{-1}$ para un control proporcional-integral-derivativo. Estos 
valores serán utilizados para las pruebas de desempeño en el banco de control de nivel.

Tabla 4. Ecuaciones para las ganancias.

\begin{tabular}{|c|c|c|c|}
\hline Control & $\mathrm{K}_{\mathrm{P}}$ & $\tau_{\mathrm{i}}[\mathrm{min}]$ & $\tau_{\mathrm{D}}\left[\mathrm{min}^{-1}\right]$ \\
\hline $\mathbf{P}$ & $\frac{1}{k}\left(\frac{t_{o}}{\tau}\right)^{-1}$ & - & - \\
\hline $\mathbf{P I}$ & $\frac{0.9}{k}\left(\frac{t_{o}}{\tau}\right)^{-1}$ & $3.33 t_{0}$ & - \\
\hline $\mathbf{P I D}$ & $\frac{1.2}{k}\left(\frac{t_{o}}{\tau}\right)^{-1}$ & $2.0 t_{0}$ & $\frac{1}{2} t_{0}$ \\
\hline
\end{tabular}

Fuente: elaboración propia, adaptada de [3].

\subsubsection{Estabilidad}

La prueba de Routh es un procedimiento para determinar el número de raíces de un polinomio con parte real positiva sin necesidad de encontrar realmente las raíces [2], [3]. El arreglo de Routh para la ecuación (53), del sistema en lazo cerrado, se presenta en la tabla 5. El coeficiente $b_{1}$ para completar la primera columna del arreglo se calcula mediante la ecuación (71).

$$
b_{1}=-\frac{\operatorname{det}\left[\begin{array}{cc}
\tau_{i}+18.72 K_{c} \tau_{D} \tau_{i} & 18.72 K_{c} \\
0.085 \tau_{i}+18.72 K_{c} \tau_{i} & 0
\end{array}\right]}{0.085 \tau_{i}+18.72 K_{c} \tau_{i}}
$$

A partir de la tabla 5 y del resultado de la ecuación (71) se establecen las condiciones para el equilibrio en las ecuaciones (72) a (73). Remplazando los valores de $\mathrm{K}_{\mathrm{c}}$, $\tau_{\mathrm{i}} \mathrm{y} \tau_{\mathrm{D}}$ de la tabla 4 para un control PID en la ecuación (53) se obtiene la ecuación (75) que cumple con las ecuaciones (72) a (73), y consecuentemente tiene los polos, $\mathrm{p}_{12}$, ubicados en el semiplano complejo con parte real negativa, en $\mathrm{p}_{12}=-5.2 \pm 1 \mathrm{j}$.

Tabla 5. Arreglo de Routh para estabilidad del sistema.

\begin{tabular}{c|c|c}
$\mathrm{s}^{2}$ & $\tau_{\mathrm{i}}+18.72 \mathrm{~K}_{\mathrm{P}} \tau_{\mathrm{D}} \tau_{\mathrm{i}}$ & $18.72 \mathrm{~K}_{\mathrm{P}}$ \\
\hline $\mathrm{s}^{1}$ & $0.085 \tau_{\mathrm{i}}+18.72 \mathrm{~K}_{\mathrm{P}} \tau_{\mathrm{i}}$ & 0 \\
\hline $\mathrm{s}^{0}$ & $\mathrm{~b}_{1}$ &
\end{tabular}

Fuente: elaboración propia.

$$
\begin{gathered}
18.72 K_{P} \geq 0 \\
\tau_{i}+18.72 K_{P} \tau_{d} \tau_{i} \geq 0 \\
0,085 \tau_{i}+18,72 K_{P} \tau_{i} \geq 0,
\end{gathered}
$$

$$
12 s^{2}+125 s+339=0
$$

\subsection{Pruebas del proceso}

Se realizaron una serie de pruebas en el banco de control de nivel para analizar, evaluar y comparar los resultados obtenidos. Se tomaron los datos para tres tipos diferentes de controladores Proporcional (P), Proporcional-Integral (PI) y Proporcional-Integral-Derivativo (PID). En la figura 16 se muestran las variables del proceso para un controlador $\mathrm{P}$, con $\mathrm{K}_{\mathrm{P}}=15$ (de la tabla 4). Similarmente, en la figura 17 se muestra cómo las variables del proceso interactúan cuando se establece un controlador PI configurado por $\mathrm{K}_{\mathrm{P}}=13.6$ y $\tau_{\mathrm{i}}=37 \mathrm{~s}$.

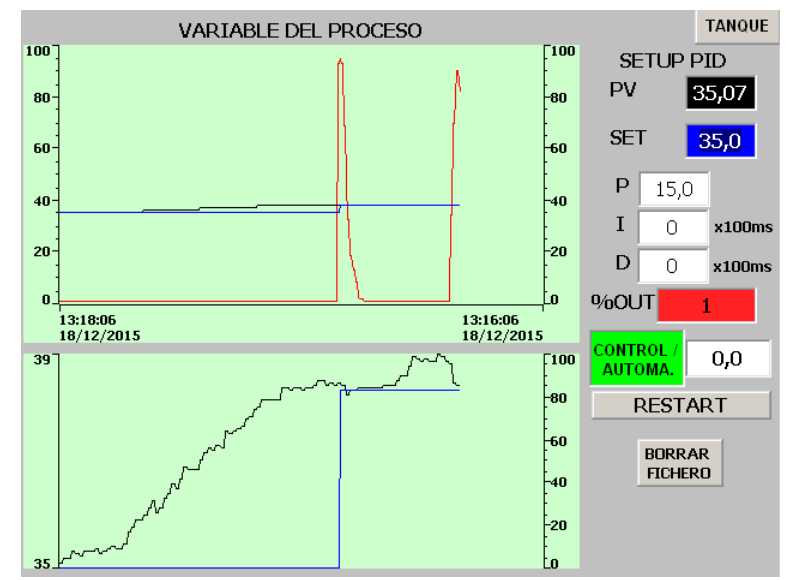

Figura 16. Salida en interfaz del proceso para un controlador proporcional. Fuente: elaboración propia.

La figura 18 muestra la salida para un controlador PID con $K_{P}=18.1, \tau_{i}=22.3 \mathrm{~s}$ y $\tau_{D}=5.6 \mathrm{~s}-1$. Se aprecia en estas figuras la configuración de las ganancias y tiempos calculados, así como la visualización del valor presente de la variable controlada y el porcentaje de salida de la válvula de control. También permite establecer el punto de referencia del nivel (set point) y cambiar entre el control manual y automático. Se presenta la visualización de las variables del proceso con respecto al tiempo en dos ventanas dentro de la interfaz. La línea azul representa el valor del set point, la línea roja es la variable de control, y representa el porcentaje de apertura de la válvula. La línea verde es la variable controlada y representa el nivel del tanque en cada instante, la ventana inferior es un zoom de la gráfica superior, mostrando ampliamente el comportamiento del nivel en el tanque. 


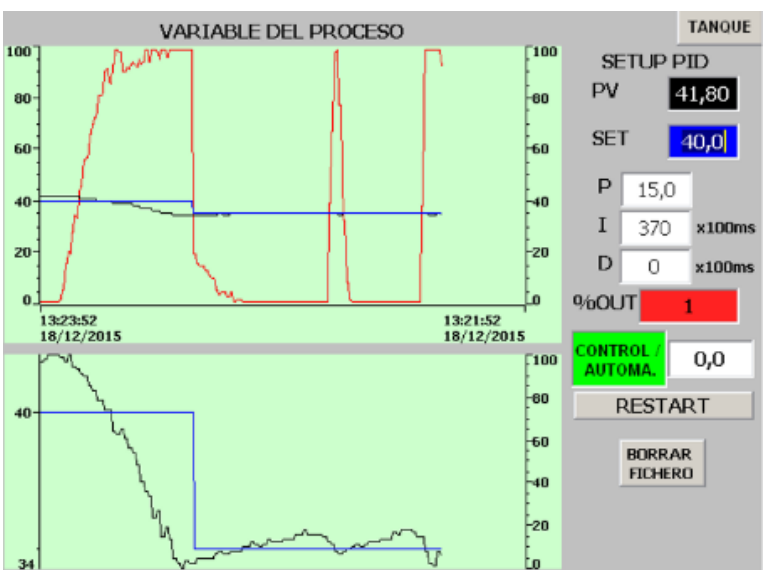

Figura 17. Salida en interfaz del proceso para un controlador proporcional-integral. Fuente: elaboración propia.

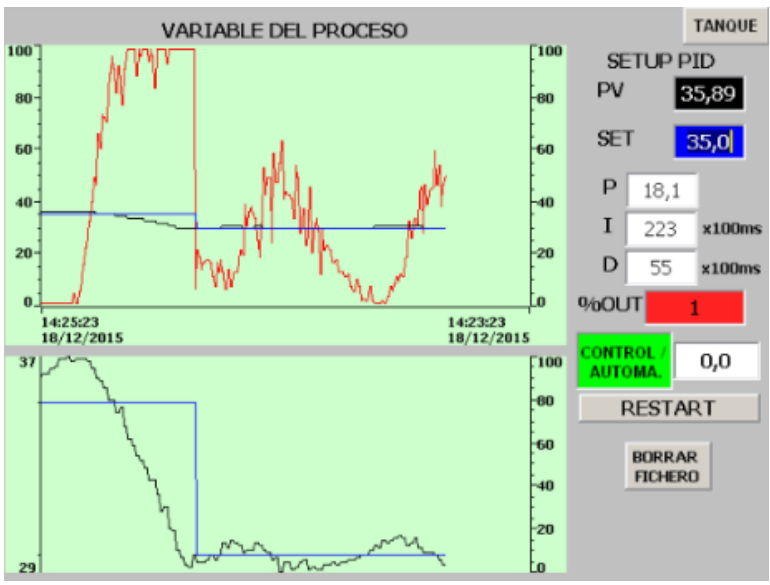

Figura 18. Salida en interfaz del proceso para un controlador proporcional-integral-derivativo. Fuente: elaboración propia.

\section{Discusión de resultados}

Con el fin de evaluar los resultados obtenidos en el banco de control de nivel, se realizaron simulaciones del proceso de nivel en Simulink, figura 19, y se obtuvo la respuesta ideal del sistema para una entrada tipo escalón, donde se genera la curva de reacción del proceso, figura 20. Se realizó el circuito de control de nivel en Simulink y se ingresaron los valores de la función de transferencia del proceso. Se introdujo al sistema un retardo de $12 \mathrm{~s}$ determinado empíricamente, con base en el tiempo que el sensor tarda en emitir la primera lectura desde que el sistema es energizado. Se estableció un nivel mínimo de $0 \mathrm{~m}$ y un nivel máximo de $40 \mathrm{~cm}$; se configuró la opción PID con sintonización del controlador realizada por la opción de Simulink (autotunning). El scope agregado permite visualizar la respuesta del sistema. La figura 20 presenta la respuesta teórica del sistema a una entrada tipo escalón. La diferencia entre la aproximación lineal y la función real es menor en las cercanías del punto de operación, y mayor cuando se aleja de este. Es difícil definir la región donde la aproximación lineal es lo suficientemente precisa como para representar la función no lineal. La figura 21 presenta un intervalo del sistema de la figura 15 .

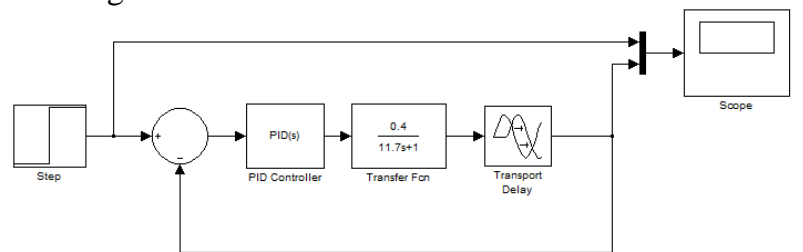

Figura 19. Circuito PID de control de nivel en Simulink. Fuente.: elaboración propia.

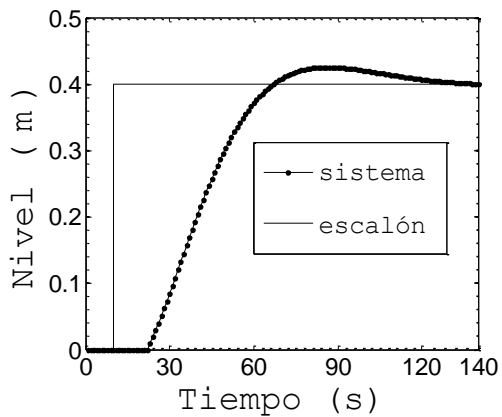

Figura 20. Respuesta teórica del sistema con controlador PID, entrada tipo escalón. Fuente: elaboración propia.

De la figura 20 se aprecia el comportamiento del sistema ideal como sistema de segundo orden subamortiguado ante una entrada tipo escalón. Su desempeño se especifica en términos de la respuesta transitoria que depende de las condiciones iniciales. Al comparar respuestas transitorias de varios sistemas, es una práctica común usar la condición inicial estándar de reposo en un tiempo inicial, por lo cual la salida y todas las derivadas con respecto al tiempo son cero.

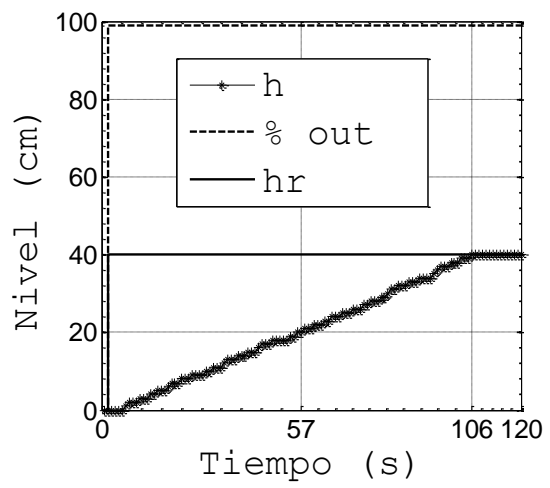

Figura 21. Respuesta teórica del modelo en Simulink con los valores de planta teórica que generan la Figura 15. Fuente: elaboración propia. 
La respuesta transitoria de un sistema real de control exhibe con frecuencia oscilaciones amortiguadas antes de alcanzar el estado estable. Al especificar las características de la respuesta transitoria de un sistema de control para una entrada escalón unitario, es común especificar lo siguiente [2], [3]:

- Tiempo de retardo, Td: tiempo requerido para que la respuesta alcance la primera vez la mitad del valor final.

- Tiempo de levantamiento, Tr: tiempo requerido para que la respuesta pase del 10 al $90 \%$, del 5 al $95 \%$, o del 0 al $100 \%$ de su valor final.

- Tiempo pico, Tp: tiempo requerido para que la respuesta alcance el primer pico del sobrepaso.

- Sobrepaso máximo (porcentaje), Mp: valor pico máximo de la curva de respuesta, medido a partir de la unidad. Si el valor final en estado estable de la respuesta es diferente de la unidad, es común usar el porcentaje de sobrepaso máximo.

- Tiempo de asentamiento, Ts: tiempo que se requiere para que la curva de respuesta alcance un rango alrededor del valor final del tamaño especificado por el porcentaje absoluto del valor final (por lo general, de 2 a $5 \%$ ) y permanezca dentro de él.

En la tabla 5, se relacionan los valores de los tiempos y niveles de los sistemas teórico y real, para los procesos de las figuras 20 y 21, respectivamente. De esta tabla se puede deducir cómo el proceso ideal es mucho más rápido que la repuesta del proceso real, para una entrada tipo escalón; el tiempo de retardo en el proceso ideal se alcanza en la mitad del tiempo del proceso real.

El tiempo de levantamiento, $\mathrm{T}_{\mathrm{s}}$, es decir, el tiempo en el cual se alcanza por primera vez el set point, también se alcanza en la mitad del tiempo con respecto al proceso real. Se puede notar que en la figura 21 no se presenta sobrepaso, es decir en el proceso real una vez que se alcanza el set point, en ningún momento se sobrepasa este valor, al menos no durante el tiempo de asentamiento, el cual se puede decir que es el único tiempo en que concuerdan los procesos debido, en gran medida, a que el proceso ideal presenta un sobrepaso del $6 \%$, que lo obliga a ajustarse nuevamente al punto de control.

Estas diferencias de tiempo en los diferentes puntos de las gráficas son debidas principalmente a los retardos de tiempo que pueden presentarse en los dispositivos empleados en la construcción del banco de control de nivel (hardware), ya que idealmente se consideran solo las ganancias de los elementos como la válvula de control y el sensor de nivel, mas no como una función de trasferencia; todo esto con el ánimo de simplificar los cálculos. El otro dispositivo que impacta directamente en el proceso es la bomba centrífuga, la cual junto con el diseño de la tubería genera retardos de tiempo que contribuyen a que se creen estas diferencias entre el proceso ideal y el real, ya que idealmente trabaja a la eficiencia máxima según la placa, además de no considerarse las pérdidas en el sistema (únicamente fueron consideradas para la selección de la bomba). A pesar de todas estas condiciones que generan las diferencias en los procesos real e ideal, se puede apreciar que no son tan significativas para hacer que el proceso se vuelva inestable, o no se alcancen los niveles de consigna deseados. Además, después del primer arranque de la planta, se puede seguir sintonizando el controlador y al haber variaciones más pequeñas en la consigna, las respuestas del sistema se vuelven más rápidas según se ajusta el controlador.

Tabla 5. Comparación de simulación y prueba escalón.

\begin{tabular}{|c|c|c|c|c|}
\hline \multirow[t]{2}{*}{ Parámetro } & \multicolumn{2}{|c|}{$\begin{array}{c}\text { Proceso simulado } \\
\text { Figura } 20\end{array}$} & \multicolumn{2}{|c|}{$\begin{array}{c}\text { Proceso prueba } \\
\text { escalón } \\
\text { Figura } 21 \\
\end{array}$} \\
\hline & Valor & Nivel $(\mathrm{cm})$ & Valor & Nivel $(\mathrm{cm})$ \\
\hline $\mathrm{T}_{\mathrm{d}}(\mathrm{s})$ & 31 & 20 & 57 & 20 \\
\hline $\mathrm{T}_{\mathrm{s}}(\mathrm{s})$ & 58 & 40 & 106 & 40 \\
\hline $\mathrm{T}_{\mathrm{p}}(\mathrm{s})$ & 76 & 42,4 & 0 & 40 \\
\hline $\mathrm{T}_{\mathrm{s}}(\mathrm{s})$ & 128 & 40 & 120 & 40 \\
\hline $\mathrm{M}_{\mathrm{p}}(\%)$ & $6 \%$ & 42,4 & $0 \%$ & 40 \\
\hline
\end{tabular}

Fuente: elaboración propia.

A continuación, se presenta un análisis para establecer el tipo de control que mejor se ajusta al sistema de control de nivel de líquido. Para ello se estableció un punto de estado estable de $35 \mathrm{~cm}$, una perturbación de aproximadamente $1 / 4$ de vuelta en la válvula de descarga, luego se ingresa un cambio en escalón en el punto de control de $5 \mathrm{~cm}$, lo cual establece el nuevo punto de control en $40 \mathrm{~cm}$. Se registraron los datos de las respuestas del sistema para controladores tipo P, PI y PID, figuras 22, 23 y 24, respectivamente. Se observa que, ante un cambio en escalón, el controlador P tuvo una respuesta de 17 s, en comparación con los 20 s y 22s que les tomó a los controladores PI y PID, respectivamente. Esto es debido a que en el sistema de posición proporcional existe una relación lineal continúa entre la variable controlada y la posición del elemento final de control. Es decir, la válvula se mueve el mismo valor por cada unidad de desviación [24], lo cual se puede comprobar en la figura 22 , donde se observa que el 
porcentaje de salida en la válvula de control es siempre el $99 \%$.

En el sistema de respuesta del controlador PI, figura 23, un cambio en la consigna dará como resultado un movimiento inmediato del índice de la señal de la válvula de control, debido a la acción proporcional; luego de un tiempo, este índice se desplazará a una velocidad lenta, debido a la acción integral, de modo que al cabo del tiempo integral habrá repetido un porcentaje del movimiento inicial provocado por la acción integral. También se aprecia que en los instantes cercanos a alcanzar la consigna, decrece el porcentaje de salida de la válvula de control.

La acción integral es conveniente emplearla cuando es preciso mantener un valor de la variable que iguale siempre al punto de consigna, para eliminar el inconveniente del offset (desviación permanente de la variable con respecto al punto de consigna) de la acción proporcional [24]. En el sistema de respuesta del controlador PID, figura 24, la acción proporcional cambia la posición de la válvula proporcionalmente a la desviación de la variable con respecto al punto de consigna. La acción proporcional mueve la válvula siguiendo fielmente los cambios en la variable controlada multiplicados por la ganancia. Un aumento de la ganancia conduce a una mayor acción proporcional y un control más rápido.

La acción integral mueve la válvula a una velocidad proporcional a la desviación con respecto al punto de consigna. Es conveniente emplearla cuando es preciso mantener un valor de la variable que iguale siempre al punto de consigna, para eliminar el inconveniente del offset (desviación permanente de la variable con respecto al punto de consigna) de la acción proporcional [24]. En el sistema de respuesta del controlador PID, la acción proporcional cambia la posición de la válvula proporcionalmente a la desviación de la variable con respecto al punto de consigna. La señal $\mathrm{P}$ (proporcional) mueve la válvula siguiendo fielmente los cambios en la variable controlada multiplicados por la ganancia. Un aumento de la ganancia conduce a una mayor acción proporcional y un control más rápido. La acción integral mueve la válvula a una velocidad proporcional a la desviación con respecto al punto de consigna.

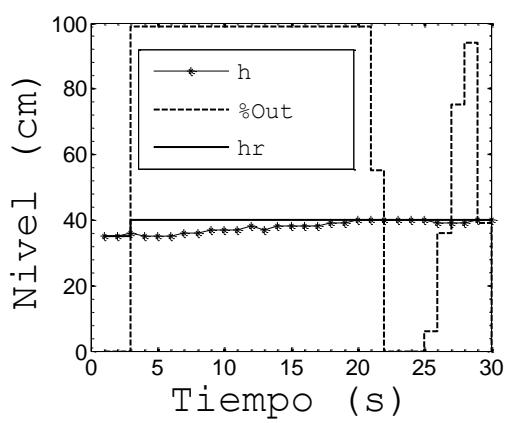

Figura 22. Respuesta del sistema ante una entrada escalón con controlador P. Fuente: elaboración propia.

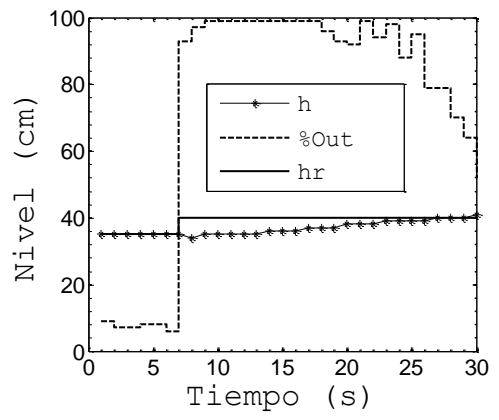

Figura 23. Respuesta del sistema ante una entrada escalón con controlador PI. Fuente: elaboración propia.

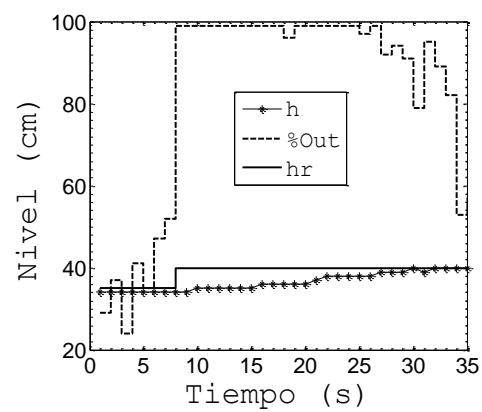

Figura 24. Respuesta del sistema ante una entrada escalón con sontrolador PID. Fuente: elaboración propia.

Finalmente, de acuerdo con las pruebas realizadas en el banco de control de nivel, se considera que un control proporcional es muy adecuado al momento de implementar un control de nivel de líquido que está sometido a pequeñas perturbaciones, dado que este tendrá la capacidad de responder ante las mismas de manera eficiente y manteniendo la variable controlada muy cerca del punto de consigna. Sin embargo, al llevar el sistema al límite, y al generar perturbaciones más grandes, sin sobrepasar las capacidades de la planta, se puede inferir que un control PI o PID tendrá mejor comportamiento dada su capacidad de eliminar el offset que se presenta en los controladores proporcionales, y a su capacidad de predecir el comportamiento del sistema mediante el cálculo de sus algoritmos de regulación. 


\section{Conclusiones}

La integración de hardware compatible desde el punto de vista funcional, compuesto por una válvula, un sensor de nivel, una bomba centrífuga, un controlador lógico programable, tuberías, accesorios de tubería y componentes eléctricos y electrónicos permitió el diseño de un circuito de proceso con la capacidad de ilustración de un proceso de control de nivel. La ecuación característica del sistema se dedujo consultando la bibliografía reconocida en el tema para el modelado del sistema, en general, y las simplificaciones correspondientes para los elementos. Esta ecuación permitió determinar que, en lazo cerrado y bajo la acción de cualquier estrategia de control tipo proporcionalintegral-derivativo, valores positivos de las ganancias garantizan la estabilidad del sistema. Se realizaron varias pruebas experimentales para recolección de datos con los cuales se determinaron valores prácticos (reales) del proceso (banco construido), tales como la constante de tiempo y los valores de las ganancias de las estrategias de control, ante una entrada tipo escalón. Mediante el uso de Simulink se modeló el sistema ideal (teórico) y su respuesta fue comparada con la experimental.

Pruebas posteriores derivaron la confrontación de resultados y discusión acerca de la acción de control más adecuada para las condiciones del proceso, de lo que se obtuvo que bajo condiciones de apreciables cambios en la consigna (set point) el tipo proporcional muestra el mejor desempeño representado en un rápido tiempo de respuesta, producto de una amplia y rápida apertura de la válvula de control. De otra parte, la implementación del tipo de control proporcional-integral-derivativo mostró ser ventajosa cuando se desea mantener la variable controlada cerca al punto de consigna, o seguir pequeños cambios del set point; la amplitud de abertura de la válvula es muy controlada y requiere un poco más de tiempo para set points relativamente grandes.

Con el desarrollo e implementación completa de esta importante y especializada herramienta didáctica, se evidencia la relación que se establece entre la teoría que se tiene de un método experimental como el de la curva de reacción del proceso y su puesta en práctica. Asimismo, se muestra lo ventajoso que resulta el aplicarlo a procesos cuyos grados de precisión son aceptables en comparación con métodos analíticos que se basan en determinar el modelo o ecuación relativa a la dinámica del sistema, y que son generalmente difíciles de aplicar por la complejidad de los procesos industriales.

El trabajo presentado se complementó con guías detalladas y videos demostrativos del desarrollo de las prácticas de laboratorio, que permitan a los estudiantes la familiarización paulatina con el equipo, normas de seguridad y el apropiado uso del banco para la implementación de una estrategia tipo PID para control de nivel. Se recomienda la consulta de este material con anterioridad al uso del banco. Se sugiere la futura implementación de estrategias de control diferente al PID que sean soportadas por el hardware integrado.

\section{Agradecimientos}

A la Vicerrectoría de Investigaciones, Extensión y Proyección Social de la Universidad del Atlántico.

\section{Referencias}

[1] Asociación Colombiana de Ingenieros Eléctricos, Mecánicos y Afines, ACIEM. Áreas de Formación de los Ingenieros, Capítulo 10: Formación Específica de los Ingenieros Mecánicos, 2006.

[2] K. Ogata, Ingeniería de control moderno, $3^{\mathrm{a}}$ ed. México: Ed Pearson, 1998, pp. 1-10.

[3] C. Smith y A. Corripio, Control automático de procesos, teoría y práctica, $1^{\mathrm{a}}$ ed. México: Ed Limusa, 1991, pp. 1-418.

[4] V. Alfaro, "Actualización del método de sintonización de controladores de Ziegler y Nichols", Ingeniería, vol. 15, n. ${ }^{\circ} 1-2$, pp. 39-52, 2005. [PDF]. Disponible en: http://eie.ucr.ac.cr/uploads/file/documentos/pub_inv/arti culos/valfaro05A.pdf

[5] K. Aström and T. Hägglund, PID controllers: Theory, design and tuning, $2^{\mathrm{a}}$ ed. USA: Instr. Soc. Of America, 1995. [PDF]: Disponible en: https://aiecp.files.wordpress.com/2012/07/1-0-1-k-jastrom-pid-controllers-theory-design-and-tuning2ed.pdf

[6] S. Martineau et al., "Four-term bilinear PID controller applied to an industrial furnace", Control Engineering Practice, vol. 12, pp. 457-464, 2004.

[7] A. Hoshinoa et al., "Improved PID method of temperature control for adiabatic demagnetization refrigerators," Nuclear Instruments and Methods in Physics Research, vol. 558, no. 2, pp. 536-541, Mar 2006. [PDF]: Disponible en: http://www.sciencedirect.com/science/article/pii/S01689 00205024708 
[8] K. Chih-Cheng, C. Chin-Wen, and F. Rong-Fong, "The self-tuning PID control in a slider-crank mechanism system by applying particle swarm optimization approach", Mechatronics, vol. 16, pp. 513522, Dec 2013. [PDF]: Disponible en: http://www.kyu.edu.tw/93/95paper/v8/95-032.pdf

[9] E. Richmond, "Diseño y construcción de una interfaz de control de nivel, temperatura y flujo de agua en un tanque para uso en prácticas de laboratorio", Pregrado, Ing. Química, Univ. Rodrigo Facio, San José, Costa Rica, 2009.

[10] J. Miranda, “Aplicación de los algoritmos PID a un controlador lógico programable", Pregrado, Ing. Eléctrica, Universidad De Costa Rica, Costa Rica, 2004.

[11] P. Mesa, "Implementación de un PLC en un sistema de regulación PID para el control de procesos de nivel, presión, temperatura, y velocidad", Pregrado, Ing. Mecánica, Univ. Central de Venezuela, Venezuela, 2004.

[12] M. Méndez y P. Erazo, "Implementación de un banco de pruebas para el control de nivel de líquidos en el laboratorio de instrumentación industrial de la Facultad de Mecánica”, Pregrado, Ing. de Mtto., Escuela Superior Politécnica de Chimborazo, Riobamba, Ecuador, 2014.

[13] O. Ardila, "Diseño, construcción y puesta en marcha de un sistema de control multiproceso", Pregrado, Ing. Química, Universidad de los Andes, Bogotá, Colombia, 2004.

[14] M. Gómez, G. Zabala y J. Dávila, "Uso de Labview para sistemas de control en ingeniería química", DYNA, vol. 78, no. 169, pp. 150-157, Oct 2011.

[15] O. Gil y D. Robayo, "Diseño, construcción y control de un sistema de almacenamiento de liquidos de segundo orden", Tecnólogo, Tecnología Eléctrica, Univ. Tecnológica de Pereira, Pereira, Colombia, 2011.

[16] C. Peñaranda, W. Silva y E. Gómez, "Instrumentación y control de nivel para un sistema de tanques acoplados en el laboratorio de control e instrumentación de E3T-UIS”, Pregrado, Dept. de Ings. Eléct. y Electrónica, Univ. Industrial de Santander, Bucaramanga, Colombia, 2014.

[17] D. Souran et al., "A Performance Comparison of Classical PID, Type-1 and Type-2 Fuzzy Controller in a Three Tank Level Control System," RoMA-IEEE Int. Symp. on Rob. and Manuf. Automation, Kuala Lumpur, Malasya, 2014, pp. 86-91.
[18] A. Ikhlef et al., "Online PID Control of Tank Level System," EDUCON-IEEE, Abu Dhabi, United Arab Emirates, 2016, 281-284.

[19] J. Fuentes, S. Castro, B. Medina, F. Moreno, y S. Sepúlveda, "Experimentación de controladores digitales clásicos en un sistema embebido aplicado en un proceso térmico," Rev. UIS Ing., vol. 17, no. 1, pp. 81-92, 2018. DOI: https://doi.org/10.18273/revuin.v17n1-2018008

[20] Flow Equations for Sizing Control Valves, ISA75.01.01, 2012.

[21] Masoneilan control valve sizing handbookMasoneilan control valves bulletin 7(00), DRESSER, UK, 2000.

[22] V. Karade, A. Shinde, and P. Pol, "Control valve coefficiente testing," Int. Journal of Innovative Research in Techn., vol. 2, no. 6, Nov 2015.

[23] EchoPod DL10-Ultrasonic Level Transmitter, FLOWLINE, Los Alamitos, CA, USA, 2015, pp. 1-2.

[24] A. Creus, Instrumentación Industrial, 8va ed. México: Editorial Alfaomega, 2010.

[25] Proportional solenoid valves 2-way servo-operated type EV260B, DANFOSS, Nordborg, Dinamarca, 2014, pp. 1-8.

[26] Effective visual communication for graphical user interfaces, Worcester Polytechnic Institute, USA, 2006. [PDF]. Disponible en: http://web.cs.wpi.edu/ matt/courses/cs563/talks/smartin /int_design.html

[27] D. Stone, et al., User interface design and evaluation, 1ra ed. Open University Ed.: Morgan Kaufmann, 2005, pp. 1-704.

[28] Diseño de pantalla, Universidad Politécnica de Cataluña, 2010. [PDF]. Disponible en: http://www.epsevg.upc.edu/hcd/material/lecturas/pantall a.pdf 